\title{
28 Research Square \\ Identification of Hepatic Fibrosis Inhibitors Through Morphometry Analysis of Hepatic Multicellular Spheroids Models
}

Haengran Seo ( $\sim$ shr1261@ip-korea.org )

Institut Pasteur de Corée: Institut Pasteur Korea https://orcid.org/0000-0003-1207-0471

Yeonhwa Song

Institut Pasteur Korea

Jinyeong Heo

Institut Pasteur Korea

David Shum

Institut Pasteur Korea

Su-Yeon Lee

Institut Pasteur Korea

\section{Sanghwa Kim}

Institut Pasteur Korea

Minji Lee

Institut Pasteur Korea

A-Ram Kim

Institut Pasteur Korea

\section{Research}

Keywords: Liver fibrosis, Multicellular tumor spheroids (MCTSs), High-throughput screening (HTS), Retinoic acid, Forskolin

Posted Date: November 17th, 2020

DOl: https://doi.org/10.21203/rs.3.rs-105306/v1

License: (c) (i) This work is licensed under a Creative Commons Attribution 4.0 International License. Read Full License 


\section{Abstract}

Background: A chronic, local inflammatory milieu can cause tissue fibrosis that results in epithelial-tomesenchymal transition (EMT), endothelial-to-mesenchymal transition (EndMT), increased abundance of fibroblasts, and further acceleration of fibrosis.

Methods: In this study, we aimed to identify potential mechanisms and inhibitors of fibrosis using 3D model-based phenotypic screening. We established liver fibrosis models using multicellular tumor spheroids (MCTSs) composed of hepatocellular carcinoma (HCC) and stromal cells such as fibroblasts (WI38), hepatic stellate cells (LX2), and endothelial cells (HUVEC) seeded at constant ratios.

Results: Through high-throughput screening of FDA-approved drugs, we identified retinoic acid and forskolin as candidates to attenuate the compactness of MCTSs as well as inhibit the expression of ECMrelated proteins. Additionally, retinoic acid and forskolin induced reprogramming of fibroblast and cancer stem cells in the HCC microenvironment. Of interest, retinoic acid and forskolin had anti-fibrosis effects by decreasing expression of a-SMA and F-actin in LX2 cells and HUVEC cells. Moreover, when sorafenib was added along with retinoic acid and forskolin, apoptosis was increased, suggesting that anti-fibrosis drugs may improve tissue penetration to support the efficacy of anti-cancer drugs.

Conclusion: Collectively, these findings support the potential utility of morphometric analyses of hepatic multicellular spheroid models in the development of new drugs with novel mechanisms for the treatment of hepatic fibrosis and HCCs.

\section{Background}

Hepatic fibrosis results as a consequence of a pattern of severe inflammation that leads to the excessive accumulation of extracellular matrix (ECM) proteins. Advanced liver fibrosis results in cirrhosis and is directly related to the high mortality of cirrhosis [1]. Because liver transplantation is currently the only treatment option for patients with advanced liver fibrosis and cirrhosis, there is an urgent need for the development of effective anti-fibrotic agents for the treatment of hepatic fibrosis.

Indeed, despite the high prevalence of liver fibrosis, there are no approved therapies, potentially because liver fibrosis represents a diverse state with numerous potential causes and complications. The main causes of liver fibrosis are chronic hepatitis virus infection[2], alcohol abuse[3], drug-induced liver injury (DILI)[4], cholestasis[5], and non-alcoholic steatohepatitis (NASH)[6]. These causal pathways are related in that they each contribute to a sustained pattern of hepatic injury and inflammation, which eventually contributes to the development of fibrotic tissue.

There are four basic cell types that reside in the liver. The specialized parenchymal cells are the hepatocytes, and the non-parenchymal cell types are principally liver sinusoidal endothelial cells (ECs), kupffer cells, and hepatic stellate cells (HCSs) [7]. Liver tissues with severe fibrosis suffer from sustained hepatocyte damage and the resulting production of fibrogenic cytokines [8] (such as TGF- $\beta 1$, angiotensin 
II), which induce the activation of non-parenchymal cells such as hepatic stellate cells [9] and ECs [10]. Activation of HSCs $[11,12]$ and endothelial-to-mesenchymal transition (EndMT) of ECs $[10,13]$ leads to deposition of uncommonly large amounts of ECM that contributes to liver fibrosis. To slow or reverse fibrosis, prior work in the development of anti-fibrotic drugs has aimed to inhibit the production of fibrogenic cytokines by reprograming of activated HCSs and/or ECs and to prevent the deposition of ECM proteins. However, there remains a strong need for the development of sophisticated in vitro methodologies that are reflective of the complex microenvironment observed in liver fibrosis to identify candidate anti-fibrotic drugs for advancement.

In our previous work, we reported the reciprocal crosstalk between cancer cells and stromal cells (fibroblast, vascular endothelial cells, and hepatic stellate cells) in a spheroid model system, which increases the expression of ECM molecules and the expression of proteins related to both epithelialmesenchymal transition (EMT) and EndMT in multicellular tumor spheroids (MCTSs) relative to monolayer culture systems. Additionally, we found that the reciprocal crosstalk between parenchymal cells and non-parenchymal cells in the spheroid system more efficiently induces transformation of HSCs and EC cells than a similar monolayer culture system $[9,14,15]$. 3D multicellular spheroids are inherently better able to capture the elements of heterogeneity, hypoxia, nutrient penetration, $\mathrm{pH}$, and metabolite gradients observed in vivo, and have thus been increasingly used as a model of cell proliferation and transformed animal models $[16,17]$. We hypothesized that the MCTSs model would be an appropriate system to mimic the behavior of the EMT, EndMT and the liver fibrosis in vivo. We have developed an automated imaging platform that systematically analyzes dynamics in cell culture based on a state-ofthe-art fluorescence imaging platform and high-end image analysis technology [18, 19].

In this study, we applied MCTSs for high-throughput screening (HTS) to identify compounds that may be able to treat liver fibrosis. To reflect extensive fibrosis in vitro, human HCC cells were grown together with human fibroblasts (WI38), human HSCs (LX2), and human umbilical endothelial cells (HUVEC) in MCTSs. Subsequently, we performed HTS with FDA-approved drugs to identify compounds that specifically reversed the fibrotic properties in MCTSs.

\section{Materials And Methods Cell culture}

Huh7 and SNU449 were purchased from the Korean Cell Line Bank (Seoul, Korea), and the human immortalized hepatocyte Fa2N-4 cell line were obtained from Xenotech (Lenexa, KS, USA). Among stromal cells, LX2 human hepatic stellate cells (HSCs) was purchased from Merck Millipore (Darmstadt, Germany), WI38 human fibroblast cell line was purchased from ATCC (Manassas, VA, USA), and human umbilical vein endothelial cells (HUVECs) were obtained from PromoCells (Heidelberg, Germany). One of the HCC cell lines, HepG2, was purchased from ATCC. All cells were maintained at $37^{\circ} \mathrm{C}$ in a humidified atmosphere of $5 \% \mathrm{CO}_{2}$. $\mathrm{HCC}$ cell lines, Huh7 and SNU449, were cultured in Roswell Park Memorial Institute medium (RPMI 1640; Welgene, Daegu, Korea) supplemented with heat-inactivated $10 \%$ fetal 
bovine serum (FBS; Gibco, Grand Island, NY, USA) and $1 \times$ penicillin-streptomycin (P/S; Gibco). HepG2 and WI38 cells were cultured in minimum essential media (MEM; Welgene) supplemented with heat inactivated 10\% FBS and 1x P/S. LX2 cells were cultured in Dulbecco's modified Eagle's medium (DMEM; Welgene) supplemented with heat-inactivated $2 \%$ FBS and 1x P/S. For HUVEC cultivation, endothelial basal medium was purchased from PromoCells. Fa2N-4 cells were plated in collagen-coated plates (BD Biosciences, San Jose, CA, USA) in serum-containing plating medium (Xenotech), and after cell attachment (approximately 3-6 hours), serum-containing plating medium was replaced with supporting culture medium (Xenotech).

\section{Generation of HCC tumor spheroids and multicellular tumor spheroids (MCTSs)}

To generate HCC tumor spheroids, cells were seeded at a density of $6 \times 10^{3}$ cells/well in 96-well round bottom ultra-low attachment microplates (Corning Life Sciences, Amsterdam, Netherlands). For MCTS generation, HCC cells were seeded at a density of $3.3 \times 10^{3}$ cells/well, and LX2, WI38, and HUVEC cells were seeded at a density of $0.9 \times 10^{3}$ cells/well/cell line together in 96-well round bottom ultra-low attachment microplates. The plates were incubated for 3 days at $37^{\circ} \mathrm{C}$ in a humidified atmosphere of $5 \%$ $\mathrm{CO}_{2}$. After 3 days, anti-fibrosis drugs were added and incubated for an additional 2 days. For MCHS generation, Fa2N-4 cells were seeded at a density of $0.9 \times 10^{3}$ cells/well/cell line together in $96-$ well round bottom ultra-low attachment microplates. The plates were incubated for 3 days at $37^{\circ} \mathrm{C}$ in a humidified atmosphere of $5 \% \mathrm{CO}_{2}$. After 3 days, anti-fibrosis drugs were added and incubated for an additional 2 days.

\section{Microarray analysis}

Global gene expression analysis was performed using Affymetrix GeneChip® Human Gene 2.0 ST Arrays. Total RNA from HCC spheroids and MCTS was isolated using the RNeasy Mini kit (Qiagen, Hilden, Germany). RNA quality was assessed using an Agilent 2100 Bioanalyser using the RNA 6000 Nano Chip (Agilent Technologies), and the quantity was determined using a Nanodrop-1000 Spectrophotometer (Thermo Fisher Scientific). We used $300 \mu \mathrm{g}$ of each RNA sample as input for the Affymetrix procedure, as recommended in the manufacturer's protocol (http://www.affymetrix.com). Briefly, $300 \mathrm{ng}$ of total RNA from each sample was converted to double-stranded CDNA using a random hexamer incorporating a T7 promoter, and amplified RNA (cRNA) was generated from the double-stranded cDNA template though an in vitro transcription (IVT) reaction and purified using the Affymetrix sample cleanup module. cDNA was regenerated through randomly primed reverse transcription using a dNTP mix containing dUTP. The cDNA was then fragmented by uracil-DNA glycosylase (UDG) and apurinic/apyrimidinic endonuclease (APE 1) restriction enzymes, and end-labeled via a terminal transferase reaction incorporating a biotinylated dideoxynucleotide. Fragmented end-labeled cDNA was hybridized to the GeneChip ${ }^{\circledR}$ Human Gene 2.0 ST array for $17 \mathrm{~h}$ at $45^{\circ} \mathrm{C}$ and $60 \mathrm{rpm}$, as described in the Gene Chip Whole Transcript (WT) Sense Target Labeling Assay Manual (Affymetrix). After hybridization, the chips were stained and washed in a Genechip Fluidics Station 450 (Affymetrix) and scanned using a Genechip Array scanner 3000 7G 
(Affymetrix). The expression intensity data were extracted from the scanned images using Affymetrix Command Console software, version 1.1, and stored as CEL files.

\section{Polyacrylamide gel electrophoresis (PAGE) and western blot analysis}

Proteins in cells were harvested in lysis buffer (Thermo Fisher Sciences, Waltham, MA, USA) through incubating for $30 \mathrm{~min}$ in ice and centrifuged at $13,200 \mathrm{rpm}$ for $10 \mathrm{~min}$ at $4{ }^{\circ} \mathrm{C}$. The supernatant was transferred to new $1.5 \mathrm{ml}$ tube (Corning, Corning, NY, USA), and the samples were mixed with $5 \mathrm{x}$ sample buffer (BioSolution, Seoul, Korea). After boiling for 10 min, equal same amounts of protein (10$30 \mu \mathrm{g} /$ well) were separated on 8 or $10 \%$ SDS-PAGE gels depending on the protein size. After electrophoresis, the proteins were transferred onto a nitrocellulose (NC) membrane (Pall, Port Washington, NY, USA), and blocked with 5\% skim milk (BD Bioscience) for 30 min at R.T. After blocking, the NC membranes were incubated with rabbit anti-human N-cadherin (ab76057, 1:500; Abcam, Cambridge, UK), rabbit anti-human E-cadherin (ab40772, 1:200; Abcam), rabbit anti-human snail (3879s, 1:1000; Cell Signaling Technology, Danvers, MA, USA), mouse anti-human vimentin (ab8978, 1:3000; Abcam), rabbit anti-human alpha smooth muscle actin (a-SMA, ab32575, 1:3000; Abcam), rabbit anti-human collagen I (NB600-408, 1:1000; Novus Biologicals, Centennial, CO, USA), rabbit anti-human Smad2/3 (3102, 1:1000; Cell Signaling Technology), rabbit anti-human p-Smad2 (Ser465/467) (3108, 1:1000; Cell Signaling Technology), rabbit anti-human p-Smad3 (Ser423/425) (9520, 1:1000; Cell Signaling Technology), mouse anti-human CD31 (3528, 1:1000; Cell Signaling Technology), mouse anti-human CD133/1 (AC133, 130090-422, 1:100; Miltenyi Biotec, Bergisch Gladbach, Germany), rabbit anti-human fibroblast activation protein (FAP, ab28244, 1:1000; Abcam), rabbit anti-human cleaved caspase-3 (ab2302, 1:1000; Abcam), and mouse anti-human $\beta$-actin (A5441, 1:10,000; Sigma-Aldrich, St Louis, MO, USA) for $16 \mathrm{hr}$ at $4{ }^{\circ} \mathrm{C}$. After washing, the blots were incubated with anti-mouse IgG, horseradish peroxidase-conjugated secondary antibody (7076, 1:10,000; Cell Signaling Technology, Danvers, MA, USA) or anti-rabbit IgG, horseradish peroxidase-conjugated secondary antibody (7074, 1:10,000; Cell Signaling Technology) for $1 \mathrm{hr}$. After washing, the specific bands were visualized by enhanced chemiluminescence (ECL; Thermo Fisher Scientific). $\beta$-actin was used as control of each samples.

\section{High-throughput screening}

A library of 4,763 compounds was assembled from Tocris Bioscience (Avonmouth, Bristol, UK), Selleck Chemicals /8Houston, TX, USA), LOPAC (St Louis, MO, USA), and Prestwich Chemical (Washington, DC, USA) and screened in the MCTS model at a final concentration of $10 \mu \mathrm{M}$ in $0.5 \%$ DMSO (v/v) (SigmaAldrich,). HCC cells and stromal cells were seeded at a density of $6 \times 103$ cells/well into 96-well round bottom ultra-low attachment microplates. To facilitate cell aggregation, the plate with the cell suspensions were centrifuged at 1,000 rpm for 1 minute before incubation. The plates were then incubated at $37^{\circ} \mathrm{C}$ in a humidified atmosphere of $5 \% \mathrm{CO}_{2}$ for 3 days. For the testing of compounds, a 2$\mu \mathrm{L}$ sample of each compound was transferred into an intermediate 384-well polypropylene plate (Greiner Bio-one, Monroe, NC, USA) using a liquid handler (Apricot Personal Pipettor; Apricot Design, Covina, CA, USA). The compounds were mixed with $78 \mu \mathrm{L}$ complete medium per well. Subsequently, a $20-\mu \mathrm{L}$ sample 
of each compound was dispensed into each well of a 96-well assay plate. The plates were then incubated at $37{ }^{\circ} \mathrm{C}$ in a humidified atmosphere of $5 \% \mathrm{CO}_{2}$ for 2 days. Controls were added to each assay plate with sorafenib at its $\mathrm{IC}_{50}$ concentration as a low control and $0.5 \% \mathrm{DMSO}(\mathrm{v} / \mathrm{v})$ as a high control. After 7 days, spheroid images were acquired using a high-content screening system. The size of spheroids was measured using a self-developed algorithm. Hit compounds were selected using a threshold based on $3 \sigma$ (standard deviation) from the $\mathrm{IC}_{50}$ of sorafenib.

\section{Staining of MCTSs}

After generating MCTSs for 3days, spheroids were treated with 5uM Retinoic acid (Sigma-Aldrich) or 5uM Forskolin (Santa Cruz Biotechnology, Dallas, TX, USA) for additional 2 days, spheroids were collected and transferred to 384-well microplates (781091, Greiner Bio-one). Spheroids were fixed with 4\%

paraformaldehyde (PFA, Biosesang, Seoul, Korea) for 30 min at R.T. After washing with DPBS for 3 times, $10 \mathrm{ul} / \mathrm{ml}$ of Alexa Fluor 488 phalloidin (A12379, Invitrogen, Eugene, OR, USA) for staining F-actin was added and incubation for $1 \mathrm{hr}$ at R.T. in dark. After washing with DPBS for 3 times, $3 \mu \mathrm{l} / \mathrm{ml}$ Hoechst33342 (H3570; Invitrogen) for staining nucleus, was added and incubation for 30 min at R.T. in dark. After washing with DPBS with 3 times, image acquisition was performed using Operetta CLS system (PerkinElmer, Waltham, MA, USA).

\section{Anti-fibrosis compound validation 2D model}

Hepatic stellate cells (LX2) or Endothelial cells (HUVECs) were seeded at $2.5 \times 103$ cells/well in 384-well microplate and incubation for $16 \mathrm{hr}$ at $37^{\circ} \mathrm{C}$ in a humidified atmosphere of $5 \% \mathrm{CO}_{2}$. LX2 and HUVECs were activated with $20 \mathrm{ng} / \mathrm{ml}$ recombinant human TGF- $\beta 1$ (100-21C, Peprotech, Cranbury, NJ, USA), and compounds were treated together for $48 \mathrm{hr}$. After incubation, cells were fixed with $4 \%$ PFA for 10 min at R.T. and reacted with rabbit anti-human a-SMA (ab32575, 1:3000; Abcam) for $16 \mathrm{hr}$ at $4{ }^{\circ} \mathrm{C}$ and washed with DPBS for $10 \mathrm{~min}$, and were incubated for $2 \mathrm{hr}$ at R.T. with goat anti-rabbit Alexa Fluor 488 secondary antibody (A11008, 1:500; Invitrogen) and Alexa Fluor Phalloidin 633 (A22284, 1:100; Invitrogen). After three times washing with DPBS, cells were stained with Hoechst 33342 for detecting nucleus. Cell images were obtained with Operetta CLS system and analyzed by Harmony software (Perkin-Elmer).

\section{Doxorubicin penetration in multicellular tumor spheroids (MCTSs)}

MCTSs were generated for 3 days, and $5 \mu \mathrm{M}$ Retinoic acid or $5 \mu \mathrm{M}$ Forskolin were incubated for 2 days. After incubation MCTSs were treated with $10 \mu \mathrm{M}$ Doxorubicin (Sigma-Aldrich) for 0, 2, or 8hr. For the image acquisition, Operetta CLS was used with a 530 to $560 \mathrm{~nm}$ excitation and a 573 to $647 \mathrm{~nm}$ emission filter set.

\section{Compounds}

Forskolin (SC-3562, Santa Cruz Technology), Retinoic acid (H7779, Sigma-Aldrich), Pirfenidone (P2116, Sigma-Aldrich), Nintedanib (S1010, Selleck Chemical), Pregnenolone succinate (700142P, Sigma-Aldrich), 
Rosiglitazone (R2408, Sigma-Aldrich), Ursocholanic acid (C7628, Sigma-Aldrich), Sorafenib (sc-220125, Santa Cruz Technology), AM580 (A8843, Sigma-Aldrich), TTNPB (S4627, Selleck Chemical), NKH477 (N3290, Sigma-Aldrich), Irsogladine maleate (sc-201190, Santa Cruz Technology), Pomiferin (CFN93047, Chemfaces), Tretinoin (PHR1187-3X, Sigam-Aldrich), Tazarotene (23559, Cayman Chemical, Ann Arbor, MI, USA)

\section{Statistical analysis}

All experiments were performed in duplicate. The results are expressed as the mean \pm standard derivation (SD). Statistical analysis was performed using Student's $t$-test.

\section{Results}

Establishment of multicellular tumor spheroids (MCTSs) that recapitulate important elements of hepatic fibrosis for high-throughput screening of potential liver fibrosis inhibitors

In our previous study, we found that the interaction between HCC cells and various non-parenchymal cells affected the compactness of the spheroids as well as cell migration through accumulation of collagen and EMT-related proteins [11]. In order to generate a fibrosis model in vitro, various HCC cell lines (Huh7 cells, SNU449 cells, and HepG2 cells) were grown together with fibroblasts (WI38), hepatic stellate cells (LX2), and endothelial cells (HUVEC) in MCTS models. Despite the fact that both SNU449 cells and HepG2 cells innately formed loose aggregates, these cells acquired the rigidness of the spheroids following co-culture with stromal cells in spheroids. Similarly, although Huh7 cells formed a relatively solid spheroids, co-culture with stromal cells enhanced rigidness in spheroids [Figure 1-A]. This result showed that crosstalk between stromal cells and HCC cells in MCTS models was an important determinant of rigidness of spheroids, emphasizing the importance of culturing these cells as a system rather than as individual components.

Next, gene expression profiling was performed on the MCTS model systems to compare against the expression profiles observed in tumor spheroids. In the MCTS models, genes that were involved in the production of ECM structural constituents were significantly enriched. In particular, we found increased relative expression of $M M P 1, C O L 6 A 1, C O L 6 A 3$, and TGFB1 in MCTS relative to tumor spheroids [Figure $1-B]$.

Because the process of EMT leads to organ fibrosis, we compared the expression of mesenchymal markers such as vimentin, a-smooth muscle actin (a-SMA), Snail, and N-cadherin between the MCTS models and tumor spheroids. Mesenchymal markers were generally upregulated in MCTS models relative to tumor spheroids alone. Because TGF- $\beta 1$, which is a critical regulator of fibrosis, stimulates the EMT and EndMT processes through the activation of Smad, we measured the expression and activation of Smad2 and Smad3. Expression of Smad2/3 and relative abundance of p-Smad2, p-Smad3 were upregulated in Huh7-, HepG2-MCTSs. SNU449 MCTSs also displayed upregulated p-Smad3 expression [Figure 1-C, Supplementary Fig. 1]. 
The MCTSs provided a useful in vitro model of liver fibrosis, where increased size of spheroids, which results from the loss of tight cross-linking among cells, indicates decreased expression of fibrosis-related proteins and thus provides a reliable morphometric indication of the reversal of liver fibrosis [Figure 1-D]. Next, we sought to establish a MCTS-based drug screening platform for the evaluation of potential liver fibrosis inhibitors. To obtain reproducible results, we plated single, homogenously sized and configured spheroids in 384-well plates for HTS screening.

A library comprised of 4,763 drug compounds with known molecular targets was tested for potentially promising candidates as inhibitors of fibrosis. All compounds were screened at an initial concentration of $10 \mu \mathrm{M}$ in duplicate to confirm the reproducibility of the observed effects. A correlation coefficient of 0.89 for replicate screens indicated that the assay was reliable [Figure 1-E]. In that screening, we identified 12 positive compounds (HITs) including four compounds involved in the cAMP/PKA pathway, five retinoic acid analogs, an anti-diabetic drug, a regulator of cholesterol, and a NMDA receptor modulator [Table I].

Because nintedanib and pirfenidone were recently authorized for the treatment of idiopathic pulmonary fibrosis, we evaluated the effects of both drugs on the size of HCC-MCTSs. Surprisingly, nintedanib and pirfenidone did not alter the morphology of HCC-MCTSs relative to control solvent ( $2 \%$ DMSO) [Supplementary Fig. 2]. On the other hand, treatment with $10 \mu \mathrm{M}$ concentration of the 12 HITs significantly increased of size of HCC-MCTSs, according to morphometric analyses, suggesting inhibition of fibrosis.

Next, dose-response studies were performed to find the most effective compounds among the 12 HITs identified from HTS of HCC-MCTSs. We found that the retinoic acid analogs and modulators of CAMP/PKA pathway, particularly retinoic acid and forskolin, led to the most significant increases of HCCMCTSs at concentrations as low as $0.1 \mu \mathrm{M}$, in a dose-dependent manner [Figure 1-F, Supplementary Fig. 3].

\section{Retinoic acid and forskolin reversed EMT and EndMT in stromal cells in multicellular tumor spheroids (MCTSs), but not multicellular hepatocyte spheroids (MCHSs)}

Generally, hepatic fibrosis is associated with upregulated expression of a-SMA via EMT and EndMT. To investigate the architectural changes observed in MCTSs following treatment with retinoic acid and forskolin, we used immunohistochemistry to evaluate the expression of F-actin in spheroid structures. Interestingly, when $5 \mu \mathrm{M}$ of retinoic acid and forskolin were added to MCTSs for $48 \mathrm{hr}$, spheroid size was bigger than DMSO-treated MCTSs, without increasing of cell size, demonstrating loss of tight cell-cell interactions and decreasing F-actin intensity among the cells [Figure 2-A].

Western blot analysis also showed that elevation of a-SMA expression in MCTSs was sufficiently attenuated by treatment with $1 \mu \mathrm{M}$ retinoic acid and forskolin, whereas expression of CD31 was increased under the same conditions. These results indicated that retinoic acid and forskolin inhibit the EndMT process as well as fibrotic properties in MCTSs [Figure 2-B, Supplementary Fig. 4]. The retinoic acid analogs AM580 and TTNPB and the water-soluble forskolin derivative NKH477 also inhibited a-SMA 
expression in MCTSs, but did not alter expression of CD31, in contrast to retinoic acid and forskolin in MCTSs [Supplementary Fig. 5-A].

Upregulation of CD133 facilitates EMT in various cancers [20-22]. Interestingly, expression of CD133 in MCTSs was also inhibited by treatment with $1 \mu \mathrm{M}$ retinoic acid and forskolin [Figure 2-C]. However, AM580, TTNPB and NKH477 did not inhibit CD133 expression as effectively as retinoic acid and forskolin [Supplementary Fig. 5-B].

Next, we were curious whether the replacement of HCC cells with normal hepatocytes in the MCHSs would result in the same phenotypic effects. Instead of Huh7, we used Fa2N-4, a well-known normal hepatocyte cell line, to generate MCHSs. As shown in Fig. 2-D, when stromal cells were mixed together, they formed compact spheroids. Similar to MCTSs, MCHSs also showed increasing expression of vimentin, a-SMA, collagen I, and Snail as well as decreasing E-cadherin and CD31 as was observed in the MCHS models [Figure 2-E, Supplementary Fig. 6]. However, the hit compounds identified from HTS, retinoic acid and forskolin, did not change the size of spheroids created with normal hepatocytes. This suggests that MCTSs composed of HCCs exist in a severe inflammatory environment that is treatable with these compounds, making it a more suitable model for screening compounds than MCHSs with normal hepatocytes [Figure 2-F].

Liver fibrosis is a complex phenomenon orchestrated by numerous cellular actors in tumor microenvironments. These results suggested that retinoic acid and forskolin may inhibit hepatic fibrosis through reversing EMT and EndMT processes of stromal cells in MCTSs and suggested that an MCTS model-based morphometric screening approach may be a good strategy for the screening of novel effective therapies for fibrosis.

\section{Retinoic acid and forskolin depolarized hepatic stellate cells in a fibrotic environment}

To confirm the potential efficacy of retinoic acid and forskolin in reprogramming activated HSCs, which are the main collagen-producing cells in liver fibrogenesis, we conducted cellular phenotype-based assays. Increasing production of a-SMA $[23,24]$ and F-actin stress fibers are associated with HSC activation when HSCs are stimulated with TGF- $\beta 1$. To define distinctive morphometric signatures before and after TGF- $\beta 1$ treatment, we focused on the expression pattern of F-actin and a-SMA after treatment with TGF- $\beta 1$. Treatment with TGF- $\beta 1$ increased the intense cytoplasmic $\alpha-S M A$ and F-actin of LX2 cells in a dose-dependent manner [Figure 3-A]. When the intensity of a-SMA and F-actin were analyzed by Harmony 3.5.1® high-content imaging and analysis software, we found that treatment with $5 \mathrm{ng} / \mathrm{ml} \mathrm{TGF-}$ $\beta 1$ increased the intensity of a-SMA more than 1.5-fold compared to the control, whereas intensity of Factin increased only slightly [Figure 3-B]. Therefore, we selected a-SMA as a marker of fiberized hepatic stellate cells. Western blot analysis also displayed similar results in agreement with the cellular phenotype-based assays. Expression of fibroblast markers, a-SMA, fibroblast activation protein (FAP) and collagen I were increased after TGF- $\beta 1$ treatment in LX2 cells [Figure 3-C]. 
Next, we measured the effects of retinoic acid and forskolin on TGF- $\beta 1$-induced HSC activation using cellular phenotype-based assays. As expected, $1 \mu \mathrm{M}$ retinoic acid and forskolin inhibited the expression of a-SMA after treatment with TGF- $\beta 1$ in LX2 cells, with efficacy comparable to $10 \mu \mathrm{M}$ pirfenidone, which served as our positive control [Figure 3-D]. Pirfenidone [25, 26] and nintedanib [27], which are FDAapproved anti-fibrotic drug, inhibit TGF- $\beta 1$-induced fibrogenesis. However, in our system, the intensity of a-SMA was not decreased as much as $2 \%$ FBS-treated control when pirfenidone and nintedanib were treated at various concentrations [Supplementary Fig. 7]. When $1 \mu \mathrm{M}$ retinoic acid [Figure 3-E] and forskolin [Figure 3-F] were added with TGF- $\beta 1$ to LX2 cells, EMT-related markers N-cadherin and Snail were inhibited, but E-cadherin was elevated, in contrast to EMT-related markers [Figure 3-E, F]. Collectively, this phenotypic-based 2D assay system using LX2 cells appears to be an effective tool for validating antifibrosis compounds and suggested that retinoic acid and forskolin can reprogram activated hepatic stellate cells.

\section{Retinoic acid and forskolin suppress the EndMT process in HUVEC}

In our previous studies, we established a visual phenomic screening platform to measure radiationinduced EndMT using HUVECs [28]. Herein, this technology was applied to measure TGF $\beta 1$-induced EndMT in HUVECs. HUVECs treated with TGF- $\beta 1$ expressed increasing amounts of F-actin and cytoplasmic a-SMA in a dose-dependent manner [Figure 4-A]. When the intensity of a-SMA and F-actin were analyzed by Harmony 3.5.1® high-content imaging and analysis software, we found that treatment with $20 \mathrm{ng} / \mathrm{ml}$ TGF- $\beta 1$ increased the intensity of a-SMA more than 1.8-fold compared to the control, and intensity of F-actin increased 1.6-fold compared to the control [Figure 4-B]. Expression of fibroblast marker, a-SMA was increased after TGF- $\beta 1$ treatment in HUVEC cells [Figure 4-C]. Next, we examined the effects of retinoic acid and forskolin on TGF $\beta 1$-induced HUVEC activation using the cellular phenotypebased 2D assay system. In this experiment, we found that $1 \mu \mathrm{M}$ retinoic acid and forskolin decreased the expression of a-SMA after TGF- $\beta 1$ treatment in HUVECs relative to treatment with $10 \mu \mathrm{M}$ pirfenidone [Figure 4-D]. Of interest, expression of a-SMA were inhibited when $1 \mu \mathrm{M}$ retinoic acid and forskolin were added with TGF- $\beta 1$ to HUVEC cells, [Figure 4-E, F]. These results suggested that anti-fibrotic compounds, such as retinoic acid and forskolin, suppress the EndMT process in HUVECs.

\section{The combination of anti-cancer drugs and anti-fibrosis compounds improves responses by enhancing penetration of anti-cancer drugs}

Liver cancer patients typically experience fibrosis, cirrhosis, and liver-related disease. As shown in Fig. 2-A and $F$, spheroids showed loose compactness after treatment with anti-fibrosis compounds, and cell-cell tight junction interactions were also weak compared to controls. In our previous study [11], we compared the efficacy of drug penetration by detecting the distribution of doxorubicin using fluorescence microscopy in HepG2 spheroids and HepG2-MCTS grown with LX2 or WI38 cells. In this study, we sought to determine whether the anti-fibrosis compounds may increase the penetration of anti-cancer drugs in MCTSs by decreasing cell-cell interactions. When the MCTSs that were treated with $5 \mu \mathrm{M}$ retinoic acid or 
forskolin were treated with $10 \mu \mathrm{M}$ doxorubicin for $8 \mathrm{hr}$, the distribution of doxorubicin in MCTSs was highly increased relative to spheroids that were not treated with retinoic acid or forskolin [Figure 5-A]. Indeed, doxorubicin only penetrated the periphery of MCTSs after treatment with $0.5 \%$ DMSO. This result was not surprising in light of the observed decreased compactness of MCTSs after treatment with antifibrosis compounds. Based on these results, we expected that anti-fibrosis compounds may accelerate anti-cancer effects by enabling delivery of anti-cancer compounds to the center of tumor tissues. In MCTS models, we found that spheroids treated with $1 \mu \mathrm{M}$ retinoic acid or forskolin combined with 1 or 3 $\mu \mathrm{M}$ of sorafenib had high expression of cleaved caspase-3 (an apoptosis marker), significantly higher relative to spheroids treated with sorafenib alone [Figure 5-B]. From these results, it appears that antifibrosis compounds, such as retinoic acid or forskolin, may improve the efficacy of anti-cancer drugs and attenuate tissue compactness and stiffness observed in liver fibrosis.

\section{Discussion}

Fibrosis has been identified as a key factor that influences survival in patients with non-alcoholic steatohepatitis (NASH). Hepatic fibrosis frequently progresses to cirrhosis and hepatocellular carcinoma, but it does not cause symptoms itself. Since there is currently no standard treatment for hepatic fibrosis, there is currently a strong incentive for pharmaceutical companies to develop safe and effective therapeutics. Further, because there are no therapies currently approved for the treatment of hepatic fibrosis, this disease is designated by the FDA as a Fast Track Development indication. In recent years, strategies of target-based approaches for screening small-molecules have shifted the strategic landscape in the evaluation of drugs that may treat hepatic fibrosis. FXR agonists (such as obeticholic acid) have demonstrated a dramatic reduction in progression and improvement in fibrosis in a phase 2 clinical study, but ultimately failed in phase 3 clinical study because of long-term toxicity. ASK-1(MAP3 kinase 5) inhibition with selonsertib reduced hepatic fibrosis in mouse models, but phase 3 study of selonsertib failed to reprogram hepatic fibrosis. Additionally, a C-C chemokine receptor type 2 (CCR2) and type 5 (CCR5) antagonist (cenicriviroc) provided anti-fibrotic activity in adult patients with hepatic fibrosis, but the anti-fibrotic effect did not meet the primary end point in a phase 2 clinical study. Hence, there remains a significant unmet need for safe and effective medications for the treatment of hepatic fibrosis in NASH.

Investigations of the molecular mechanisms of hepatic fibrosis have presented several clear targets such as TGF- $\beta 1$, PPAR, ASK-1, angiotensin, YAP-TEAD, various inflammatory cytokines, and ROS. However, there remains no validated target for novel anti-fibrotic compounds.

To develop the novel compounds for hepatic fibrosis, we first need to understand the complexity of the molecular mechanisms that govern hepatic fibrogenesis and the local microenvironment. Hepatic fibrosis is caused by chronic inflammation, and the liver tissue becomes rigid due ECM accumulation. Further, this environment results in EMT or EndMT activation. There have been numerous studies assessing novel drugs for fibrosis and molecular mechanisms using 2D culture systems for cells in monolayer on plastic culture dishes. However, recent evidence has suggested that 2D systems fail to capture several crucial elements of the 3D environment, and 3D culture systems may be a more effective culture method. Diverse 
phenotypic approaches for drug screening assays have become increasingly popular in drug discovery as an alternative strategy to target-based approaches for the assessment of potential treatments for hepatic fibrosis [29-31]. Particularly, 3D co-culture models represent a high-throughput phenotypic screening system to efficiently screen for new anti-fibrotic therapeutics [32-36]. In this study, we tested whether MCTS models may recapitulate the in vivo microenvironment in fibrosis to generate a phenotype-based model that could overcome the shortcomings seen with 2D systems.

In addition to abnormal HSC activation, ECM deposition and stiffness are key phenotypes observed in hepatic fibrosis in vivo. Thus, strategies to reverse HSC activation, ECM deposition, and stiffness in spheroid models are critical to develop effective therapeutic agents for fibrosis. We found that MCTSs possessed ECM structural constituents [Figure 1]. Currently, the most common agents that are prescribed off-label for hepatic fibrosis in NASH include vitamin E, ursodeoxycholic acid, pioglitazone, metformin, and lipid-modifying agents. Retinoic acid, retinoic acid analogs, and cAMP activators such as forskolin can attenuate hepatic stellate cell activation and have been validated with animal studies [37-40]. Interestingly, a series of off-label agents for hepatic fibrosis such as ursocholanic acid, rosiglitazone, and retinoic acid analogs were included among the HIT compounds identified herein [Figure 2, Table I]. Forskolin and forskolin derivatives (NKH 477) attenuate carbon tetrachloride-induced liver fibrosis in rats and were also identified as HIT compounds. The anti-fibrotic effects of forskolin have already been shown in animal models of liver fibrosis [41, 42] and intestinal organoids [36]. Hence, our MCTS-based screening system appears to represent an effective approach for the identification of future therapeutics of fibrosis, providing comparable results with animal experiments.

TGF- $\beta$ plays a key role in the progression of liver fibrosis, and drugs that inhibit TGF- $\beta$ have been shown to have anti-fibrotic effects in animal studies. The MCTS model used in this study also represented the ECM-related protein accumulation as well as p-smad activation [Figure 1]. We found that reducing the expression of CD133, a cancer stem cell marker associated with liver cancer, had anti-fibrotic effects and also regulates the surrounding environment, potentially influencing risk of HCC.

Moreover, when we developed spheroids using normal hepatocytes instead of HCC cells in MCHSs, they showed ECM accumulation or mesenchymal cell properties, but did not change the phenotypic properties after drug treatment. From this result, HCC cells with stromal cells appear to best represent pathological characteristics of hepatic fibrosis.

As mentioned earlier, 2D phenotypic assay systems have been adapted for selecting anti-fibrotic compounds through LX2 cell activation with TGF- $\beta 1$. In Fig. 3 and Fig. 4, we also utilized this system for secondary validation of hit compounds from the MCTS-based screening. Hit compounds from the screen were also effective at inhibiting endothelial activity and hepatic stellate activation. The failure rate in clinical trials could possibly be reduced by deriving the first hit through MCTS-based screens and then verifying drug efficacy in a 2D assay that can verify inhibition of EMT and EndMT, followed by confirmation in animal models of disease. 
Biopsies of tissues from HCC patients commonly show evidence of cirrhosis and NASH. It has been suggested that drug treatment efficacy in liver cancer patients is lower than other carcinomas due to the hepatic microenvironment. Tissue rigidity due to the accumulation of ECM and excessive inflammatory reactions lowers drug permeability, which reduces the ability of therapeutic compounds to access target cells. We have previously reported that losartan reduced the robustness of MCTS and consequently increased the permeability to doxorubicin. Similarly, in this work, drug permeability was increased when the anti-fibrotic drugs retinoic acid and forskolin were used to treat MCTSs [Figure 5]. The anti-cancer effect of sorafenib, a common treatment for liver cancer, was improved after combined treatment with these anti-fibrotics identified in our model system.

\section{Conclusions}

In this study, an in vitro model that reflects the microenvironment observed in hepatic fibrosis in vivo was constructed, characterized, and tested as a model for screening drugs that may be effective treatments for liver fibrosis. We expect that this model offers an efficient, high-throughput strategy to identify new drugs and targets through phenotypic screening. We found that anti-fibrotic drugs are not only effective in the treatment of liver fibrosis, but can also enhance the anti-cancer activity of other therapeutics by increasing tissue permeability, allowing drug delivery to cancer cells of interest.

\section{Abbreviations}

ASK-1

Apoptosis signal-regulating kinase 1

a-SMA

a-smooth muscle actin

CAMP

cyclic adenosine monophosphate

DILI

drug-induced liver injury

DMSO

Dimethyl sulfoxide

EC cells

Enterochromaffin cells

ECM

extracellular matrix

EndMT

endothelial-to-mesenchymal transition

EMT

epithelial-mesenchymal transition

EthD-1 
Ethdium homodimer-1

FAP

fibroblast activation protein

FDA

Food and Drug Administration

FXR

Farnesoid $\mathrm{X}$ receptor

HCC

hepatocellular carcinoma

HSCs

hepatic stellate cells

HTS

high-throughput screening

HUVEC

Human Umbilical Vein Endothelial Cells

$\mathrm{NASH}$

non-alcoholic steatohepatitis

NMDA

$N$-methyl-D-aspartate

MAP3

Mitogen Activated Protein 3

MCHSs

Multicellular hepatocyte spheroids

MCTSs

Multicellular tumor spheroids

PKA

protein kinase $A$

PPARs

peroxisome proliferator-activated receptors

ROS

Reactive oxygen species

SD

standard derivation

TEAD

Transcriptional enhancer factor TEF/ TEA domain family member

TGF- $\beta$

Trransforming growth factor-beta

TME

Tumor microenvironments

YAP 
yes-associated protein

2D

Two dimensional

3D

Three dimensional

\section{Declarations}

\section{Consent for publication}

All authors read and approved the final manuscript for publication.

\section{Availability of data and materials}

Information is included in the Methods section.

\section{Competing interests}

The authors declare that they have no competing interests.

\section{Funding}

This work was supported by the National Research foundation of Korea (NRF) grant funded by the Korea government (MSIP) (NRF-2017M3A9G7072864, NRF-2017M3A9G6068246 and NRF2019R1C1C1006374) and Gyeonggi-do

\section{Authors' contributions}

YS and YS designed the in vitro experiments, analyzed data and prepared the manuscript. SK, ML and AK performed forming of multicellular tumor spheroids for this study. JH, DS and SL were involved in drug screening to identify anti-fibrotic drugs. HS designed and was the overseer of the entire study.

\section{References}

1. Bataller R, Brenner DA. Liver fibrosis. J Clin Invest. 2005;115:209-18.

2. Weiskirchen R, Weiskirchen S, Tacke F: Recent advances in understanding liver fibrosis: bridging basic science and individualized treatment concepts. F1000Res 2018, 7.

3. Woolbright BL, Jaeschke H. Alcoholic Hepatitis: Lost in Translation. J Clin Transl Hepatol. 2018;6:89-96. 
4. Kleiner DE. Drug-induced Liver Injury: The Hepatic Pathologist's Approach. Gastroenterol Clin North Am. 2017;46:273-96.

5. Penz-Osterreicher $\mathrm{M}$, Osterreicher $\mathrm{CH}$, Trauner M. Fibrosis in autoimmune and cholestatic liver disease. Best Pract Res Clin Gastroenterol. 2011;25:245-58.

6. Brunt EM. Nonalcoholic steatohepatitis. Semin Liver Dis. 2004;24:3-20.

7. Clark AM, Wheeler SE, Taylor DP, Pillai VC, Young CL, Prantil-Baun R, Nguyen T, Stolz DB, Borenstein JT, Lauffenburger DA, et al. A microphysiological system model of therapy for liver micrometastases. Exp Biol Med (Maywood). 2014;239:1170-9.

8. Kovacs EJ, DiPietro LA. Fibrogenic cytokines and connective tissue production. FASEB J. 1994;8:854-61.

9. Zhang CY, Yuan WG, He P, Lei JH, Wang CX. Liver fibrosis and hepatic stellate cells: Etiology, pathological hallmarks and therapeutic targets. World J Gastroenterol. 2016;22:10512-22.

10. Dufton NP, Peghaire CR, Osuna-Almagro L, Raimondi C, Kalna V, Chuahan A, Webb G, Yang Y, Birdsey $\mathrm{GM}$, Lalor $\mathrm{P}$, et al. Dynamic regulation of canonical TGFbeta signalling by endothelial transcription factor ERG protects from liver fibrogenesis. Nat Commun. 2017;8:895.

11. Song Y, Kim SH, Kim KM, Choi EK, Kim J, Seo HR. Activated hepatic stellate cells play pivotal roles in hepatocellular carcinoma cell chemoresistance and migration in multicellular tumor spheroids. Sci Rep. 2016;6:36750.

12. Moreira RK. Hepatic stellate cells and liver fibrosis. Arch Pathol Lab Med. 2007;131:1728-34.

13. Pardali E, Sanchez-Duffhues G, Gomez-Puerto MC, Ten Dijke P. TGF-beta-Induced EndothelialMesenchymal Transition in Fibrotic Diseases. Int J Mol Sci 2017, 18.

14. Kim SH, Song Y, Seo HR. GSK-3beta regulates the endothelial-to-mesenchymal transition via reciprocal crosstalk between NSCLC cells and HUVECs in multicellular tumor spheroid models. J Exp Clin Cancer Res. 2019;38:46.

15. Song Y, Kim JS, Choi EK, Kim J, Kim KM, Seo HR. TGF-beta-independent CTGF induction regulates cell adhesion mediated drug resistance by increasing collagen I in HCC. Oncotarget. 2017;8:2165062.

16. Minchinton Al, Tannock IF. Drug penetration in solid tumours. Nat Rev Cancer. 2006;6:583-92.

17. Hirschhaeuser F, Menne H, Dittfeld C, West J, Mueller-Klieser W, Kunz-Schughart LA. Multicellular tumor spheroids: an underestimated tool is catching up again. J Biotechnol. 2010;148:3-15.

18. Jang JW, Song Y, Kim KM, Kim JS, Choi EK, Kim J, Seo H. Hepatocellular carcinoma-targeted drug discovery through image-based phenotypic screening in co-cultures of HCC cells with hepatocytes. BMC Cancer. 2016;16:810.

19. Song Y, Kim IK, Choi I, Kim SH, Seo HR. Oxytetracycline have the therapeutic efficiency in CD133(+) HCC population through suppression CD133 expression by decreasing of protein stability of CD133. Sci Rep. 2018;8:16100. 
20. Chen YS, Wu MJ, Huang CY, Lin SC, Chuang TH, Yu CC, Lo JF. CD133/Src axis mediates tumor initiating property and epithelial-mesenchymal transition of head and neck cancer. PLoS One. 2011;6:e28053.

21. Liu K, Hao M, Ouyang Y, Zheng J, Chen D. CD133(+) cancer stem cells promoted by VEGF accelerate the recurrence of hepatocellular carcinoma. Sci Rep. 2017;7:41499.

22. Ding Q, Miyazaki Y, Tsukasa K, Matsubara S, Yoshimitsu M, Takao S. CD133 facilitates epithelialmesenchymal transition through interaction with the ERK pathway in pancreatic cancer metastasis. Mol Cancer. 2014;13:15.

23. Hautekeete ML, Geerts A. The hepatic stellate (Ito) cell: its role in human liver disease. Virchows Arch. 1997;430:195-207.

24. Venturi C, Reding R, Quinones JA, Sokal E, Rahier J, Bueno J, Sempoux C. Relevance of activated hepatic stellate cells in predicting the development of pediatric liver allograft fibrosis. Liver Transpl. 2016;22:822-9.

25. Schaefer CJ, Ruhrmund DW, Pan L, Seiwert SD, Kossen K. Antifibrotic activities of pirfenidone in animal models. Eur Respir Rev. 2011;20:85-97.

26. Garcia L, Hernandez I, Sandoval A, Salazar A, Garcia J, Vera J, Grijalva G, Muriel P, Margolin S, Armendariz-Borunda J. Pirfenidone effectively reverses experimental liver fibrosis. J Hepatol. 2002;37:797-805.

27. Lin X, Wen J, Liu R, Gao W, Qu B, Yu M. Nintedanib inhibits TGF-beta-induced myofibroblast transdifferentiation in human Tenon's fibroblasts. Mol Vis. 2018;24:789-800.

28. Song Y, Lee SY, Kim AR, Kim S, Heo J, Shum D, Kim SH, Choi I, Lee YJ, Seo HR. Identification of radiation-induced EndMT inhibitors through cell-based phenomic screening. FEBS Open Bio. 2019;9:82-91.

29. Rehman M, Vodret S, Braga L, Guarnaccia C, Celsi F, Rossetti G, Martinelli V, Battini T, Long C, Vukusic $\mathrm{K}$, et al: High-throughput screening discovers antifibrotic properties of haloperidol by hindering myofibroblast activation. JCI Insight 2019, 4.

30. Bollong MJ, Yang B, Vergani N, Beyer BA, Chin EN, Zambaldo C, Wang D, Chatterjee AK, Lairson LL, Schultz PG. Small molecule-mediated inhibition of myofibroblast transdifferentiation for the treatment of fibrosis. Proc Natl Acad Sci U S A. 2017;114:4679-84.

31. Wang XT, Sun XJ, Li C, Liu Y, Zhang L, Li YD, Wu QH, Li SY, Li Y. Establishing a Cell-Based HighContent Screening Assay for TCM Compounds with Anti-Renal Fibrosis Effects. Evid Based Complement Alternat Med. 2018;2018:7942614.

32. Langhans SA. Three-Dimensional in Vitro Cell Culture Models in Drug Discovery and Drug Repositioning. Front Pharmacol. 2018;9:6.

33. Arai K, Eguchi T, Rahman MM, Sakamoto R, Masuda N, Nakatsura T, Calderwood SK, Kozaki K, Itoh M. A Novel High-Throughput 3D Screening System for EMT Inhibitors: A Pilot Screening Discovered the EMT Inhibitory Activity of CDK2 Inhibitor SU9516. PLoS One. 2016;11:e0162394. 
34. Wenzel C, Otto S, Prechtl S, Parczyk K, Steigemann P. A novel 3D high-content assay identifies compounds that prevent fibroblast invasion into tissue surrogates. Exp Cell Res. 2015;339:35-43.

35. Xu Q, Norman JT, Shrivastav S, Lucio-Cazana J, Kopp JB. In vitro models of TGF-beta-induced fibrosis suitable for high-throughput screening of antifibrotic agents. Am J Physiol Renal Physiol. 2007;293:F631-40.

36. Boj SF, Vonk AM, Statia M, Su J, Vries RR, Beekman JM, Clevers H. Forskolin-induced Swelling in Intestinal Organoids: An In Vitro Assay for Assessing Drug Response in Cystic Fibrosis Patients. J Vis Exp 2017.

37. Shimizu H, Tsubota T, Kanki K, Shiota G. All-trans retinoic acid ameliorates hepatic stellate cell activation via suppression of thioredoxin interacting protein expression. J Cell Physiol. 2018;233:607-16.

38. Senoo H, Wake K. Suppression of experimental hepatic fibrosis by administration of vitamin A. Lab Invest. 1985;52:182-94.

39. Parkes JG, Templeton DM. Effects of retinol and hepatocyte-conditioned medium on cultured rat hepatic stellate cells. Ann Clin Lab Sci. 2003;33:295-305.

40. Murakami K, Kaji T, Shimono R, Hayashida Y, Matsufuji H, Tsuyama S, Maezono R, Kosai K, Takamatsu $\mathrm{H}$. Therapeutic effects of vitamin A on experimental cholestatic rats with hepatic fibrosis. Pediatr Surg Int. 2011;27:863-70.

41. El-Agroudy NN, El-Naga RN, El-Razeq RA, El-Demerdash E. Forskolin, a hedgehog signalling inhibitor, attenuates carbon tetrachloride-induced liver fibrosis in rats. Br J Pharmacol. 2016;173:3248-60.

42. Pinzani M, Rombouts $\mathrm{K}$, Colagrande S. Fibrosis in chronic liver diseases: diagnosis and management. J Hepatol. 2005;42(Suppl:):22-36.

\section{Tables}


Table 1

List of HITs as anti-fibrotic compounds

\begin{tabular}{|ll|}
\hline HIT compounds & Function \\
\hline Irsogladine maleate & PDE4 inhibitor; antiulcer agent \\
\hline Forskolin & Adenylyl cyclase activator \\
\hline NKH 477 & Adenylyl cyclase activator \\
\hline Pomiferin & PDE5 inhibitor \\
\hline Retinoic acid & Endogenous retinoic acid receptor agonist. \\
\hline TTNPB & Retinoic acid analog; RAR agonist \\
\hline AM 580 & Retinoic acid agonist \\
\hline Tretinoin & all-trans retinoic acid \\
\hline Tazarotene & Retinoic acid receptor alpha (RAR-a) \\
Rosiglitazone & Anti-diabetic medication \\
\hline $\begin{array}{l}\text { Ursocholanic acid } \\
\text { Pregnenolone succinate }\end{array}$ & $\begin{array}{l}\text { cholesterol absorption } \\
\text { NMDA receptor modulator }\end{array}$ \\
\hline
\end{tabular}

Figures 

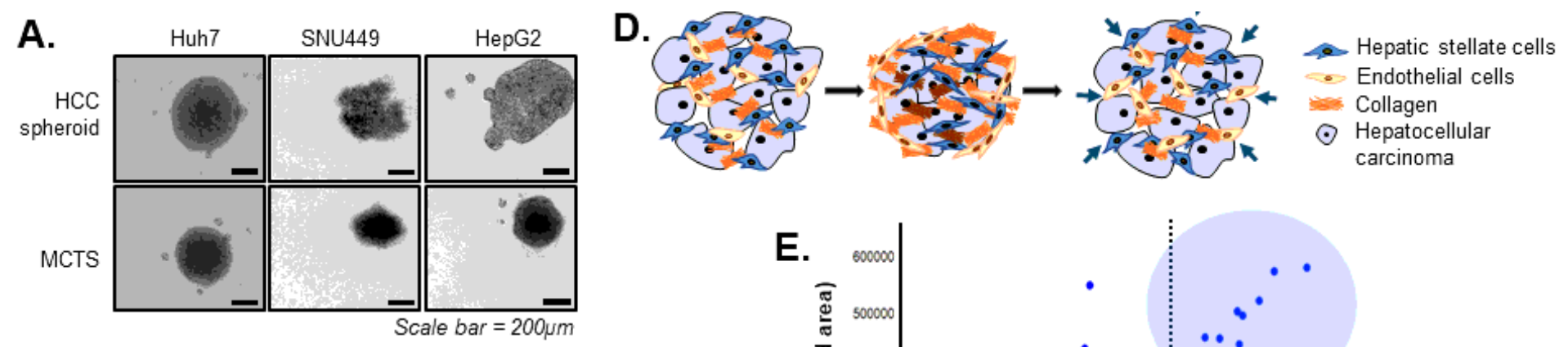

B. $\stackrel{\mathrm{HCC}}{\mathrm{Spheroid}} \mathrm{MCTS}$

C.
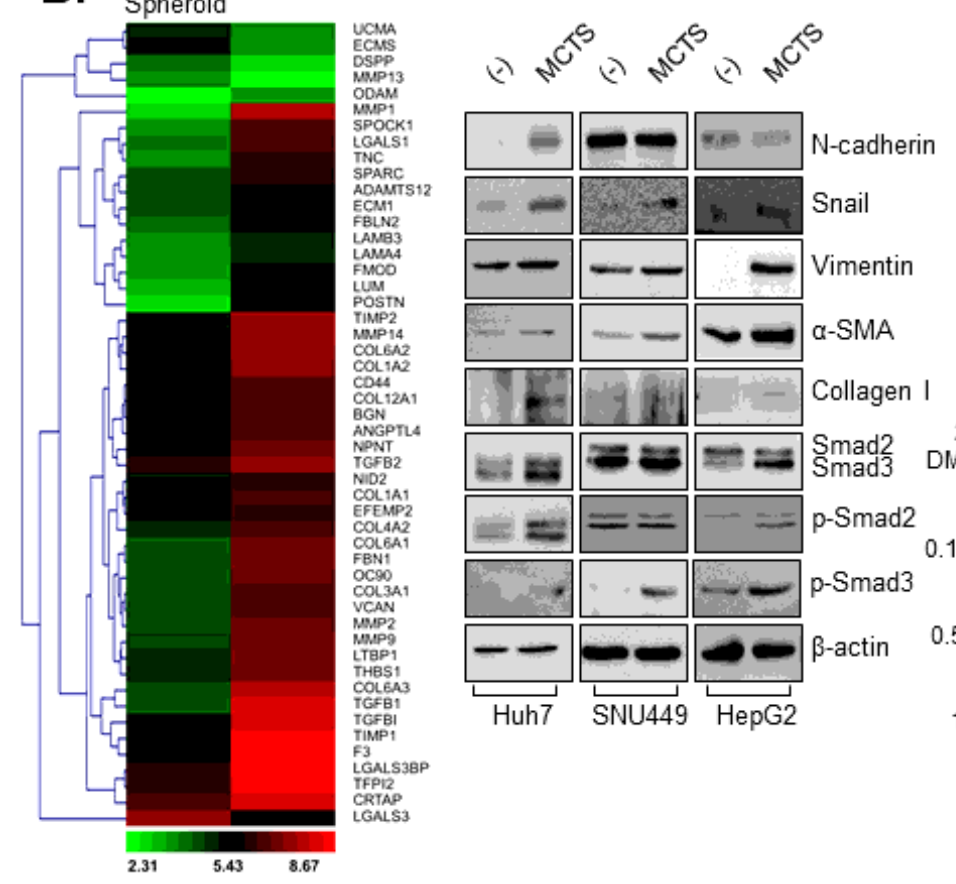

E.
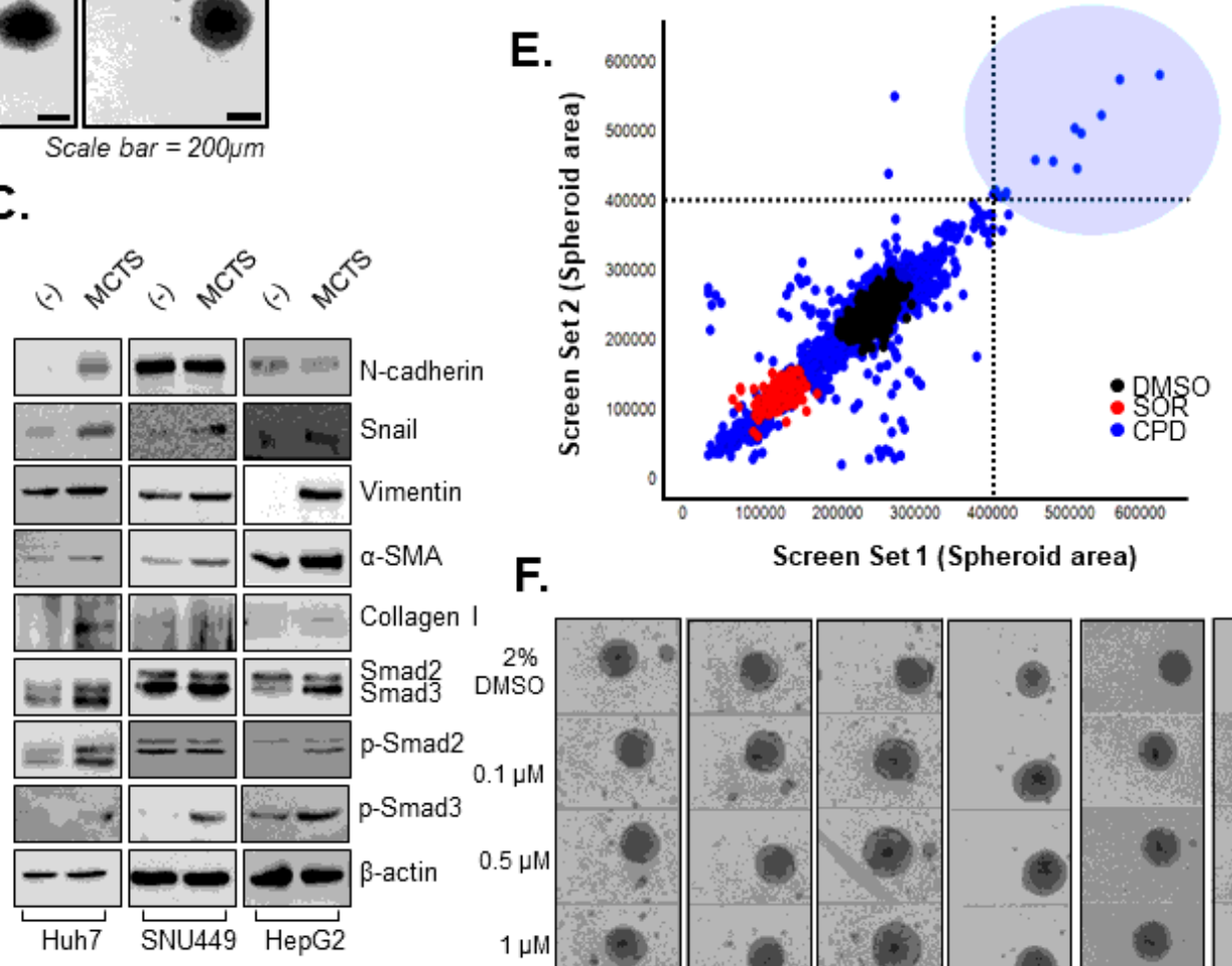

F.

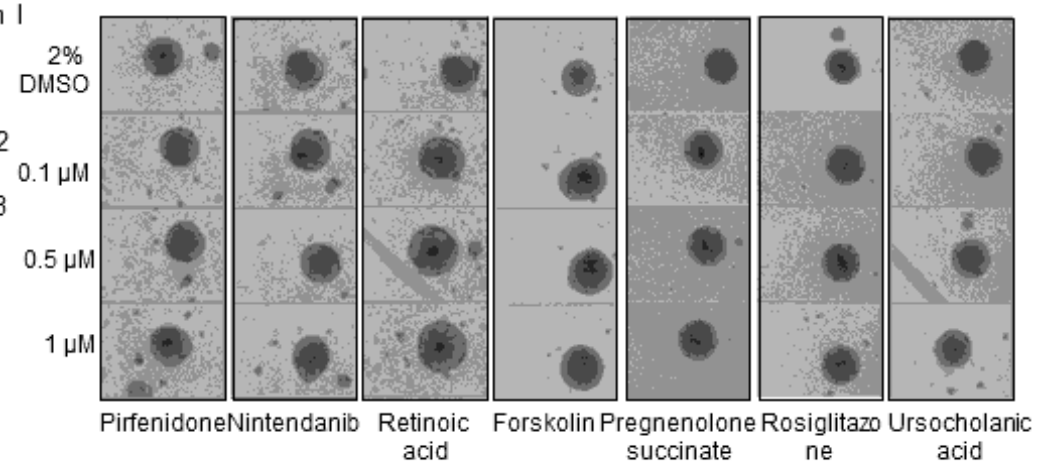

Figure 1

Establishment of multicellular tumor spheroids (MCTSs) as a model of liver fibrosis and screening for inhibitors of fibrosis. (A) HCC spheroids and MCTS were developed using an HCC cell line (Huh7, SNU449, or HepG2) and stromal cells (LX2, WI38, HUVEC). (B) Microarray analysis of Huh7 spheroid and Huh7 MCTS. (C) Mesenchymal, EMT, ECM, TGF- $\beta 1$ signaling-related protein expression in HCC spheroids and HCC-MCTSs (Huh7, SNU449, and HepG2). (D) Scheme of 3D phenomic-based assay model using MCTS in vitro. (E) The scatterplot analysis of positive (red; sorafenib IC50), negative (black: $0.5 \%$ DMSO) control, and 4,763 compounds comprised of FDA-approved drugs from a compound library. (F) MCTS treated with $0.1,0.5$, or $1 \mu \mathrm{M} \mathrm{HIT}$ compounds identified in the screen. All images were obtained using the Operetta CLS system. 

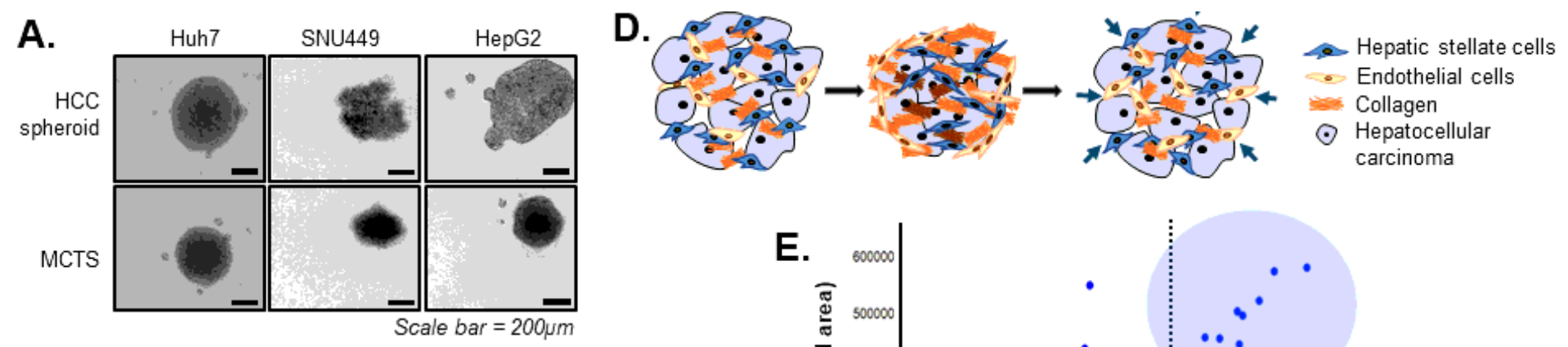

B. $\stackrel{\mathrm{HCC}}{\mathrm{Spheroid}} \mathrm{MCTS}$

C.
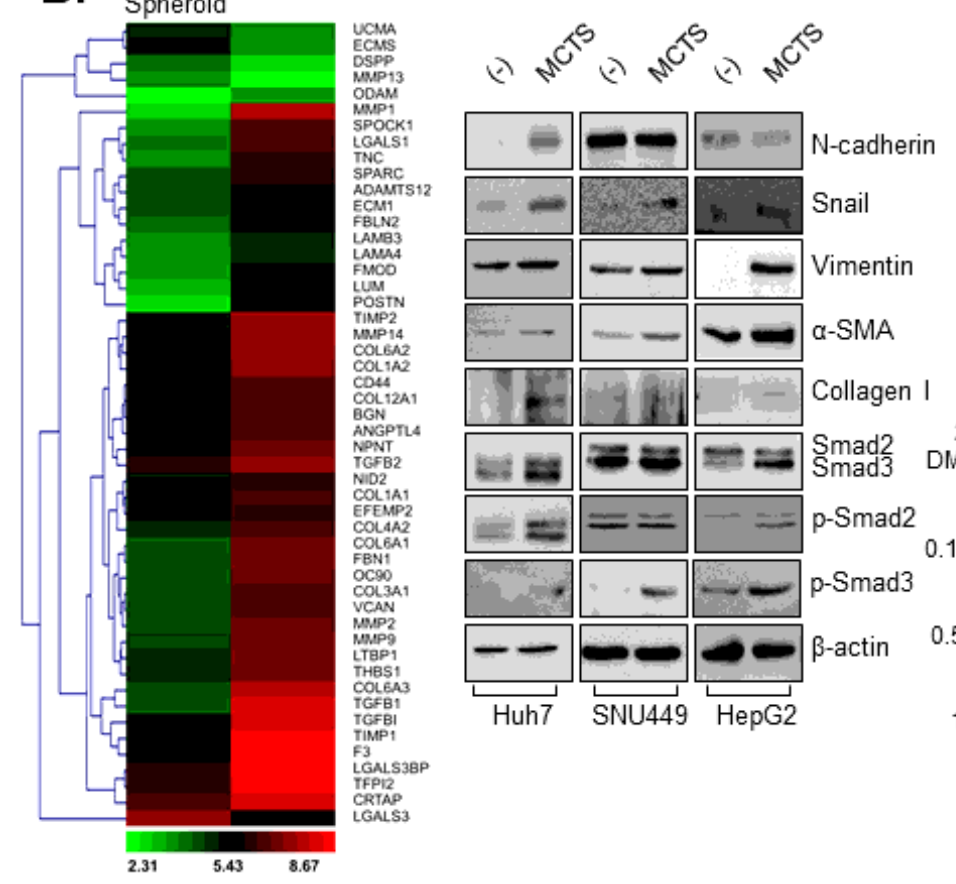

E.
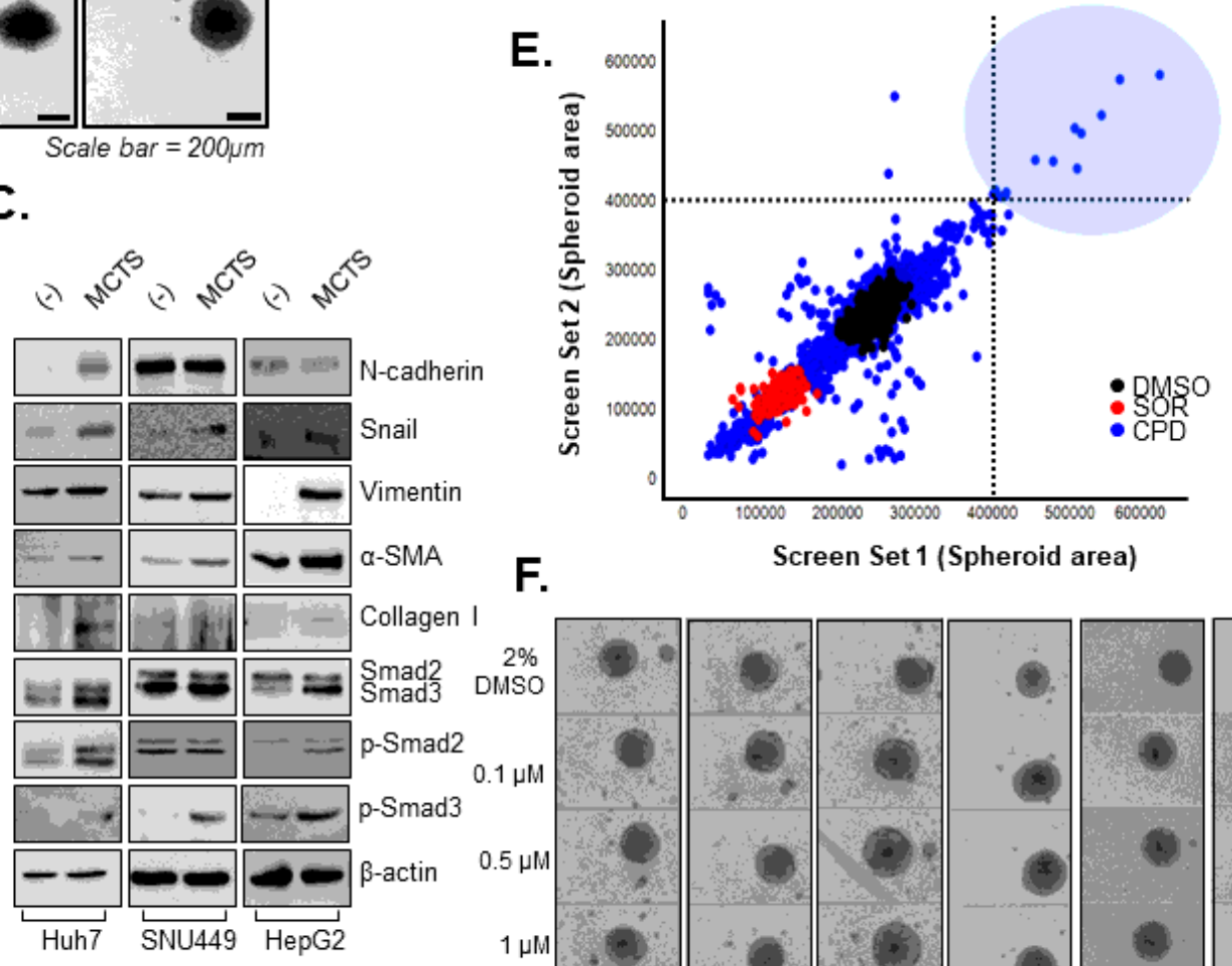

F.

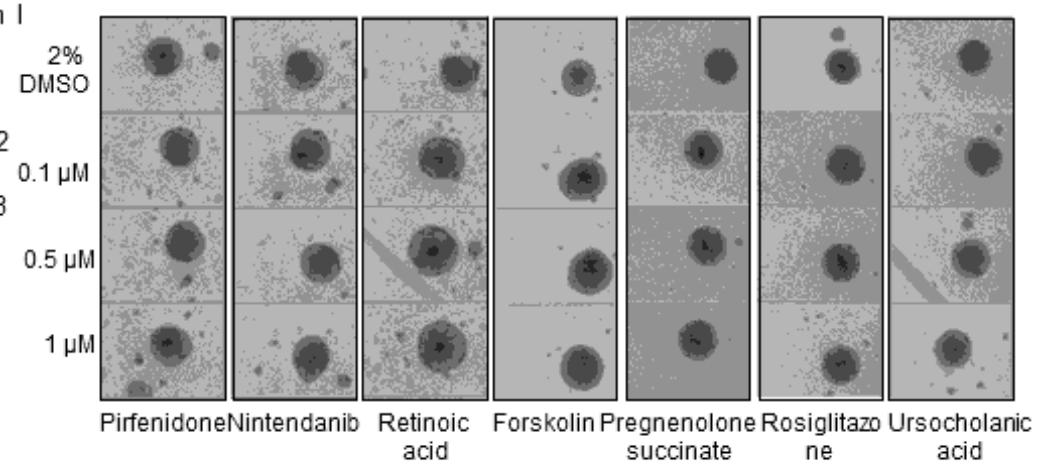

Figure 1

Establishment of multicellular tumor spheroids (MCTSs) as a model of liver fibrosis and screening for inhibitors of fibrosis. (A) HCC spheroids and MCTS were developed using an HCC cell line (Huh7, SNU449, or HepG2) and stromal cells (LX2, WI38, HUVEC). (B) Microarray analysis of Huh7 spheroid and Huh7 MCTS. (C) Mesenchymal, EMT, ECM, TGF- $\beta 1$ signaling-related protein expression in HCC spheroids and HCC-MCTSs (Huh7, SNU449, and HepG2). (D) Scheme of 3D phenomic-based assay model using MCTS in vitro. (E) The scatterplot analysis of positive (red; sorafenib IC50), negative (black: $0.5 \%$ DMSO) control, and 4,763 compounds comprised of FDA-approved drugs from a compound library. (F) MCTS treated with $0.1,0.5$, or $1 \mu \mathrm{M} \mathrm{HIT}$ compounds identified in the screen. All images were obtained using the Operetta CLS system. 

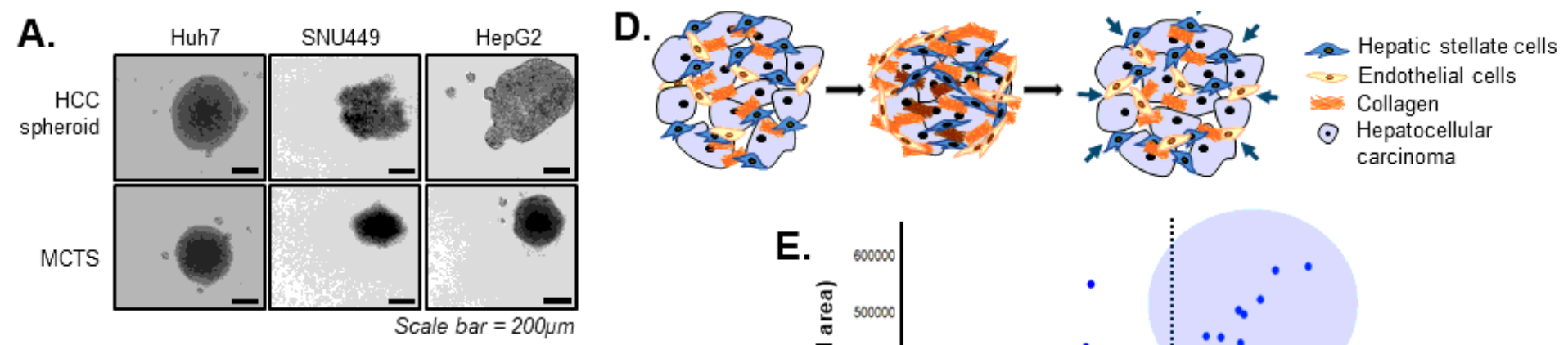

B. $\stackrel{\mathrm{HCC}}{\mathrm{Spheroid}} \mathrm{MCTS}$

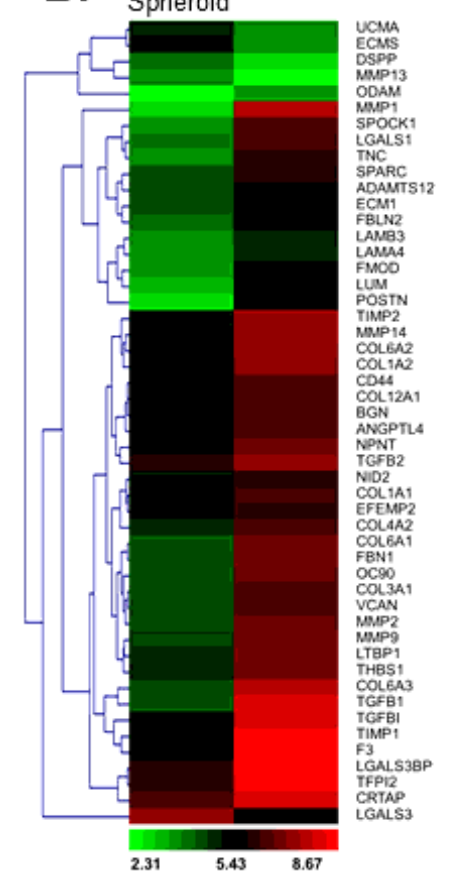

C.

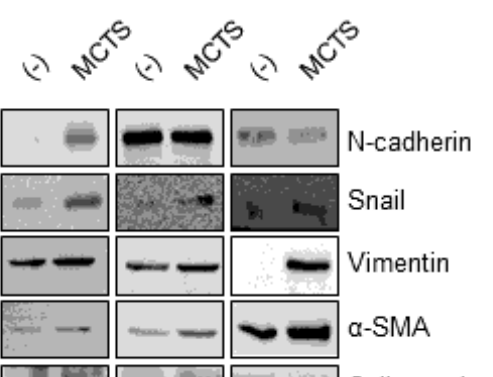

E.
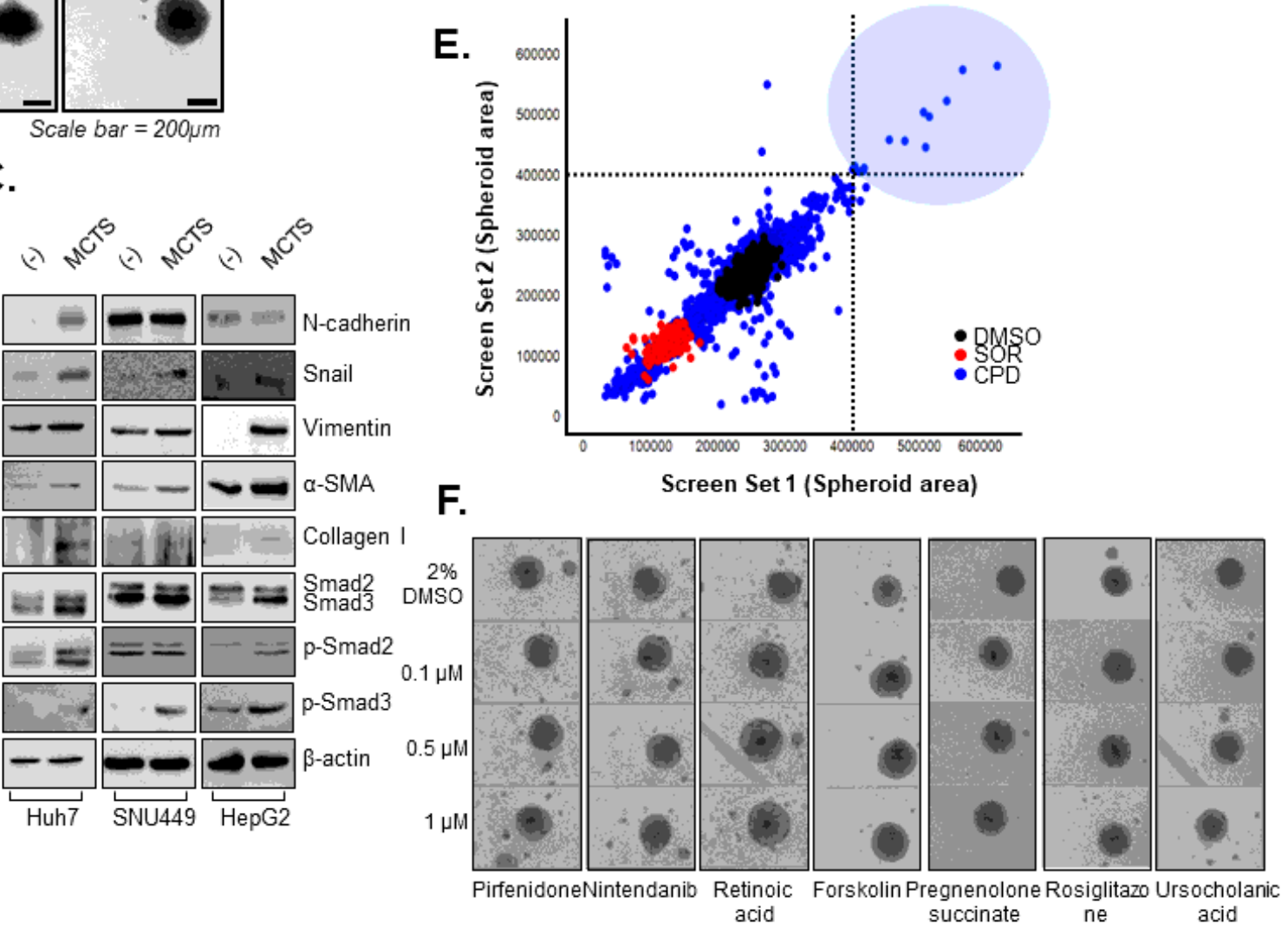

\section{Figure 1}

Establishment of multicellular tumor spheroids (MCTSs) as a model of liver fibrosis and screening for inhibitors of fibrosis. (A) HCC spheroids and MCTS were developed using an HCC cell line (Huh7, SNU449, or HepG2) and stromal cells (LX2, WI38, HUVEC). (B) Microarray analysis of Huh7 spheroid and Huh7 MCTS. (C) Mesenchymal, EMT, ECM, TGF- $\beta 1$ signaling-related protein expression in HCC spheroids and HCC-MCTSs (Huh7, SNU449, and HepG2). (D) Scheme of 3D phenomic-based assay model using MCTS in vitro. (E) The scatterplot analysis of positive (red; sorafenib IC50), negative (black: $0.5 \%$ DMSO) control, and 4,763 compounds comprised of FDA-approved drugs from a compound library. (F) MCTS treated with $0.1,0.5$, or $1 \mu \mathrm{M} \mathrm{HIT}$ compounds identified in the screen. All images were obtained using the Operetta CLS system. 
A.
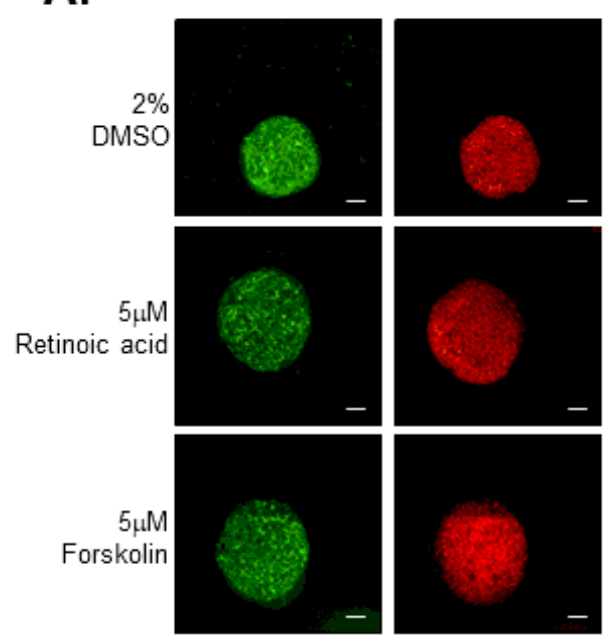

B.

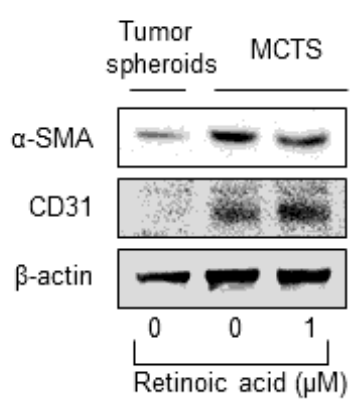

C.
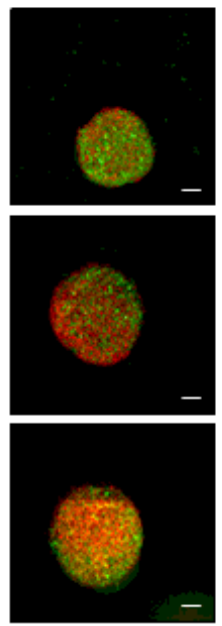

$\square$ F-actin

Nucleus

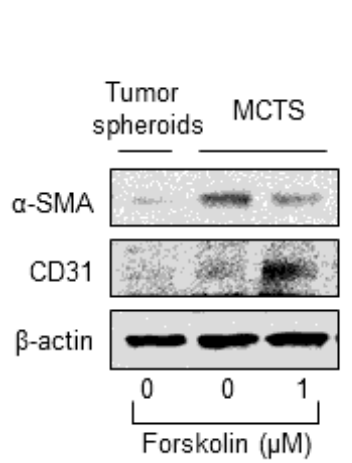

D.

E.
CD133 $\beta$-actin
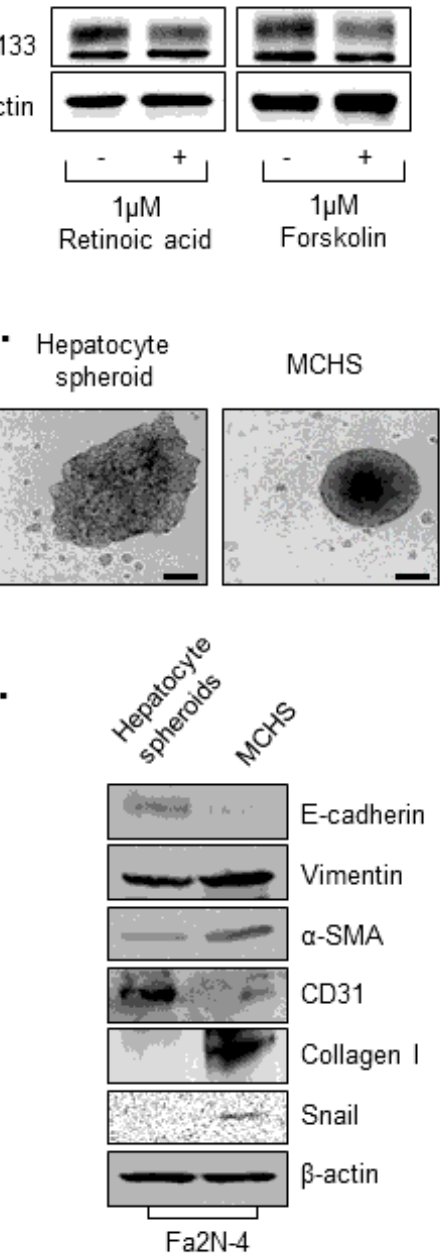

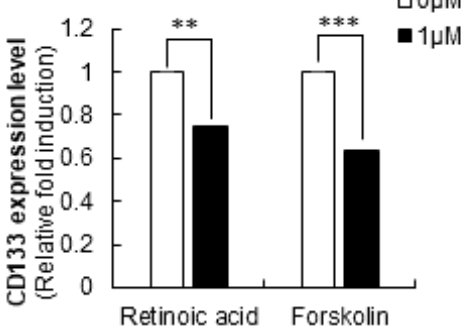

F.
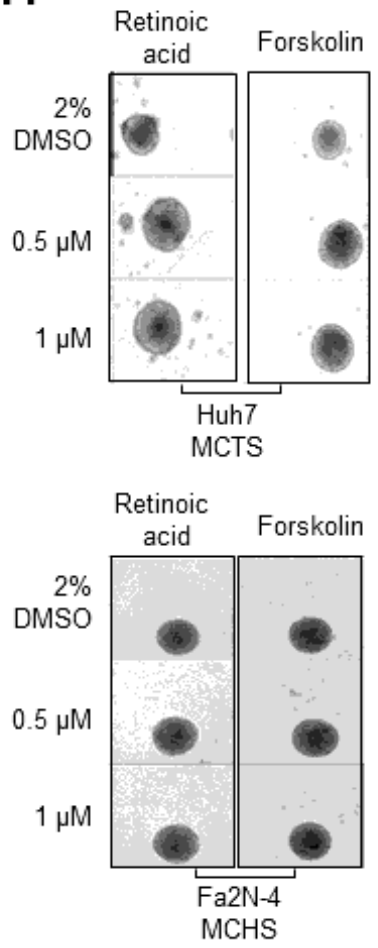

Figure 2

Reprogramming of liver fibrosis in the multicellular tumor spheroid (MCTS) model but not the multicellular hepatocyte spheroid (MCHS) model. (A) Structure through F-actin staining of MCTS treated with $5 \mu \mathrm{M}$ of retinoic acid or forskolin. (B) Expression of mesenchymal-related marker (a-SMA), and an EndMT-related marker (CD31) in tumor spheroids or MCTSs with or without treatment with $1 \mu \mathrm{M}$ retinoic acid or forskolin. (C) Expression of cancer stem cell-related marker (CD133) in MCTS with or without 1 $\mu \mathrm{M}$ retinoic acid or forskolin (left panel). The expression of CD133 was quantified (right panel). (D) Spheroid formation of Fa2N-4 spheroids or Fa2N-4 MCHSs. (E) Expression of EMT-related proteins (Ecadherin, Snail), mesenchymal-related proteins (vimentin, a-SMA), EndMT-related protein (CD31), and ECM-related protein (collagen I) in hepatocyte spheroids and MCHSs. (F) Spheroids treated with 0.5 or 1 $\mu \mathrm{M}$ retinoic acid and forskolin in MCTS and MCHS. All images were obtained using the Operetta CLS system. 
A.
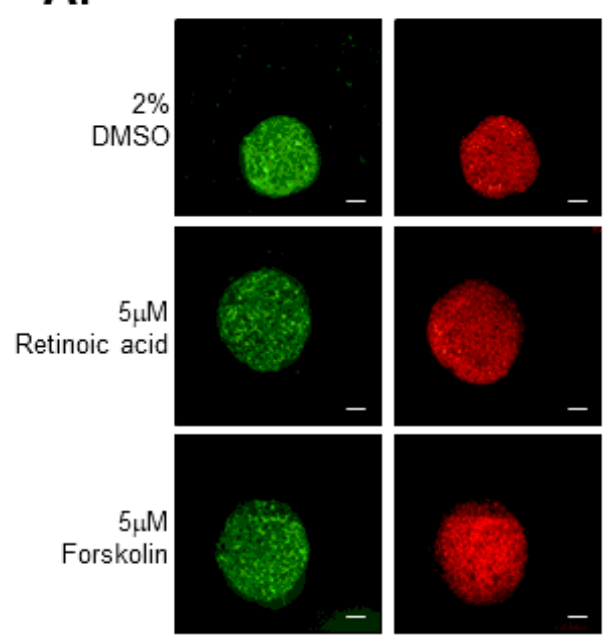

B.

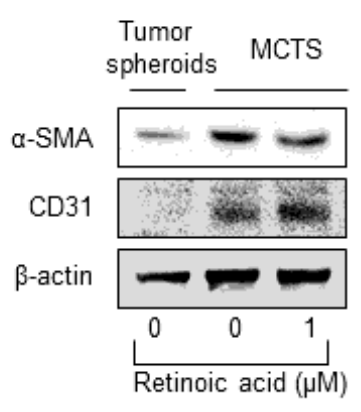

C.
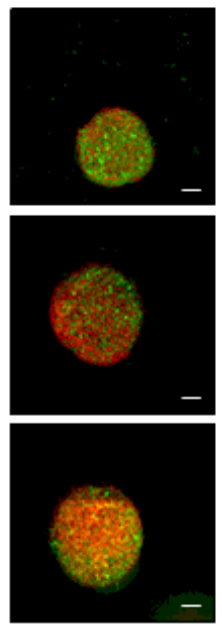

$\square$ F-actin

Nucleus

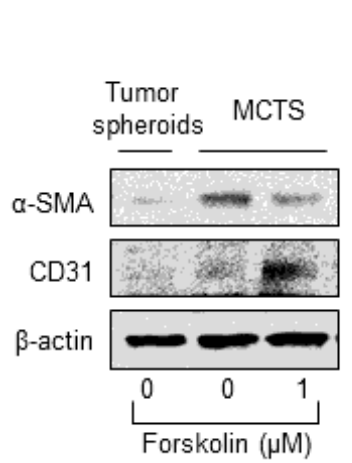

D.

E.
CD133 $\beta$-actin
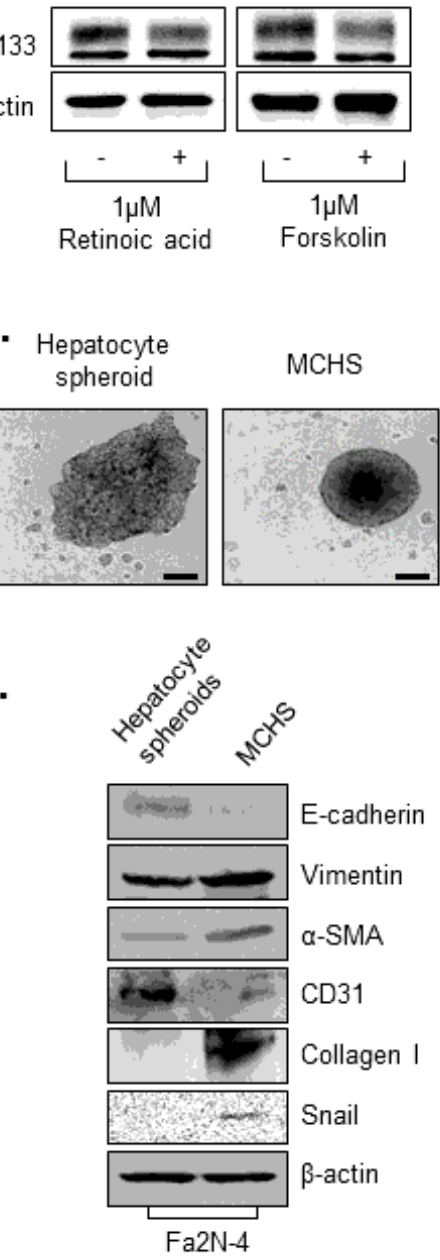

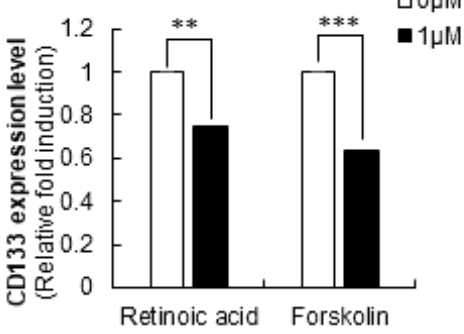

F.
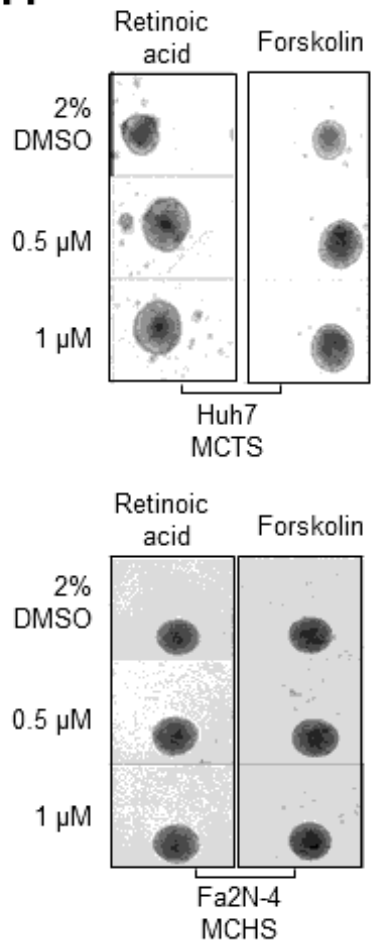

Figure 2

Reprogramming of liver fibrosis in the multicellular tumor spheroid (MCTS) model but not the multicellular hepatocyte spheroid (MCHS) model. (A) Structure through F-actin staining of MCTS treated with $5 \mu \mathrm{M}$ of retinoic acid or forskolin. (B) Expression of mesenchymal-related marker (a-SMA), and an EndMT-related marker (CD31) in tumor spheroids or MCTSs with or without treatment with $1 \mu \mathrm{M}$ retinoic acid or forskolin. (C) Expression of cancer stem cell-related marker (CD133) in MCTS with or without 1 $\mu \mathrm{M}$ retinoic acid or forskolin (left panel). The expression of CD133 was quantified (right panel). (D) Spheroid formation of Fa2N-4 spheroids or Fa2N-4 MCHSs. (E) Expression of EMT-related proteins (Ecadherin, Snail), mesenchymal-related proteins (vimentin, a-SMA), EndMT-related protein (CD31), and ECM-related protein (collagen I) in hepatocyte spheroids and MCHSs. (F) Spheroids treated with 0.5 or 1 $\mu \mathrm{M}$ retinoic acid and forskolin in MCTS and MCHS. All images were obtained using the Operetta CLS system. 
A.
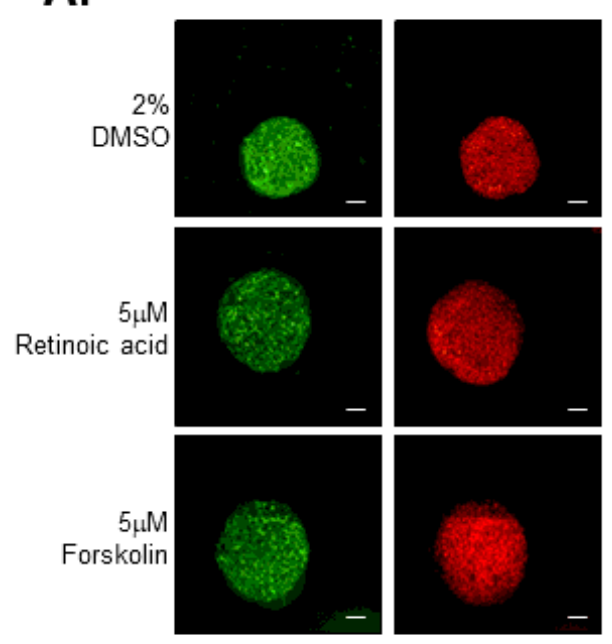

B.

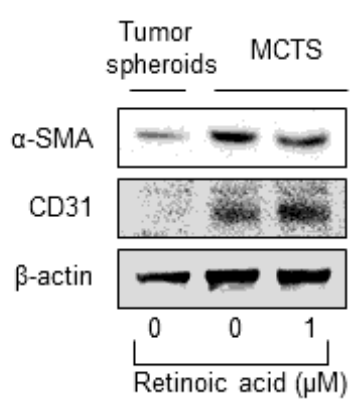

C.
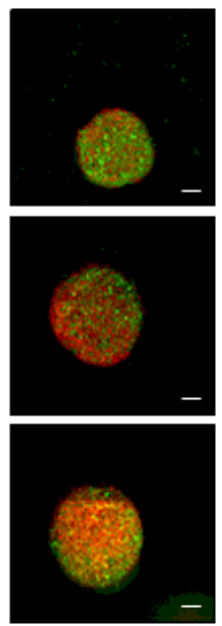

$\square$ F-actin

Nucleus

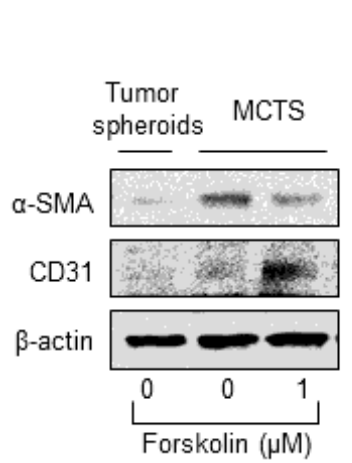

D.

E.
CD133 $\beta$-actin
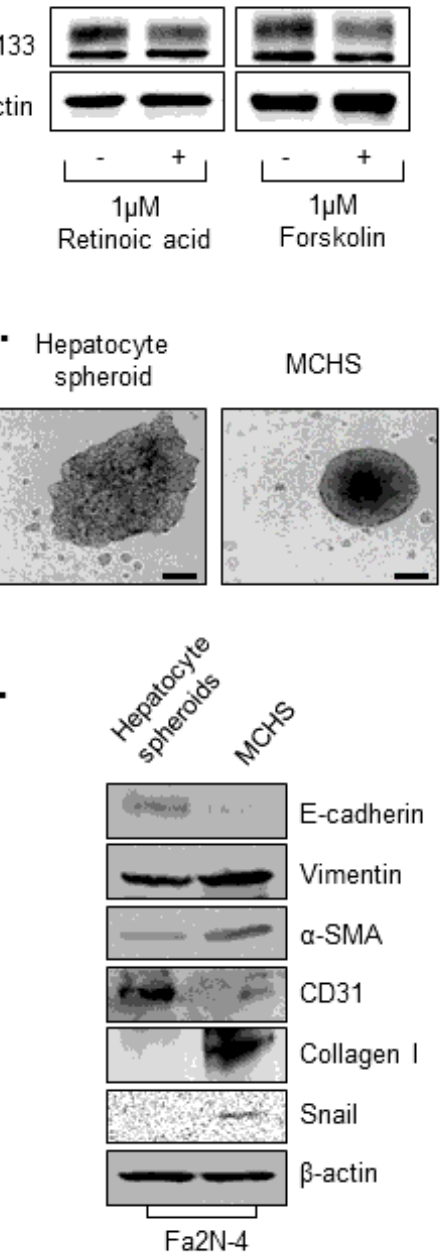

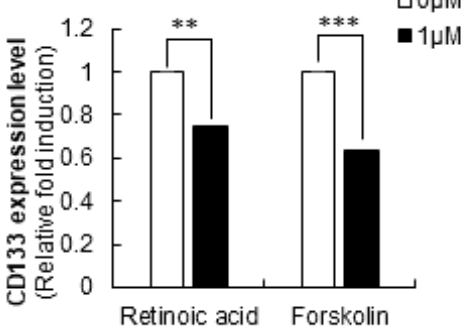

F.
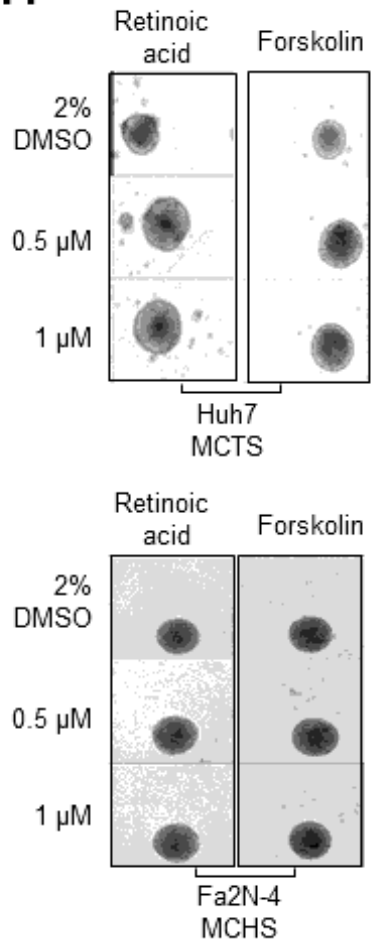

Figure 2

Reprogramming of liver fibrosis in the multicellular tumor spheroid (MCTS) model but not the multicellular hepatocyte spheroid (MCHS) model. (A) Structure through F-actin staining of MCTS treated with $5 \mu \mathrm{M}$ of retinoic acid or forskolin. (B) Expression of mesenchymal-related marker (a-SMA), and an EndMT-related marker (CD31) in tumor spheroids or MCTSs with or without treatment with $1 \mu \mathrm{M}$ retinoic acid or forskolin. (C) Expression of cancer stem cell-related marker (CD133) in MCTS with or without 1 $\mu \mathrm{M}$ retinoic acid or forskolin (left panel). The expression of CD133 was quantified (right panel). (D) Spheroid formation of Fa2N-4 spheroids or Fa2N-4 MCHSs. (E) Expression of EMT-related proteins (Ecadherin, Snail), mesenchymal-related proteins (vimentin, a-SMA), EndMT-related protein (CD31), and ECM-related protein (collagen I) in hepatocyte spheroids and MCHSs. (F) Spheroids treated with 0.5 or 1 $\mu \mathrm{M}$ retinoic acid and forskolin in MCTS and MCHS. All images were obtained using the Operetta CLS system. 

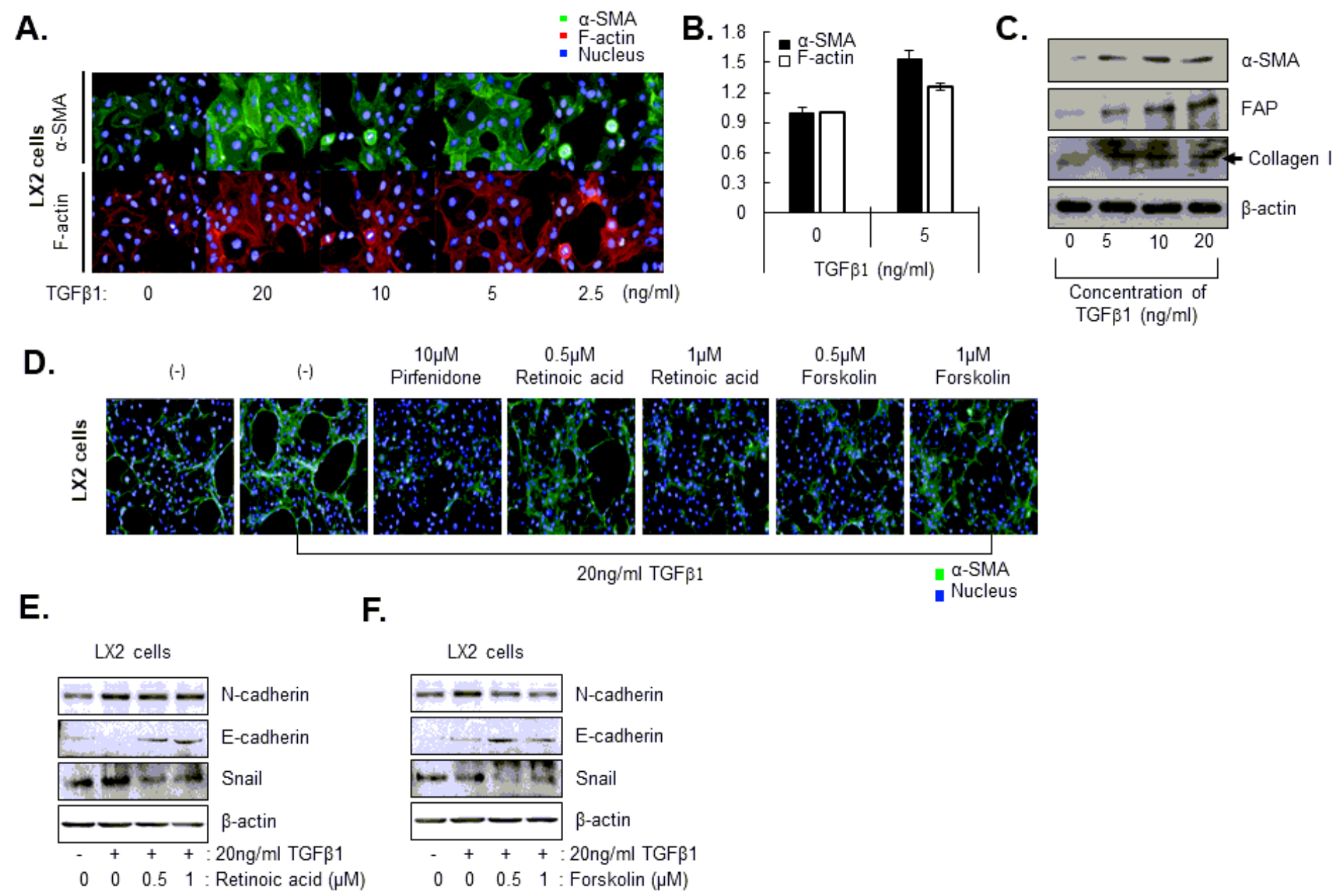

\section{Figure 3}

Anti-fibrosis effects of hit compounds in phenomic-based 2D culture of hepatic stellate cells. (A) Intensity of a-SMA and F-actin in hepatic stellate cells in a 2D culture system treated with TGF- $\beta 1$. Cells were treated with 2.5, 5, 10, or $20 \mathrm{ng} / \mathrm{ml}$ TGF- $\beta 1$ in $2 \%$ FBS media. (B) Calculated intensity of a-SMA and Factin when LX2 cells were treated with $5 \mathrm{ng} / \mathrm{ml}$ TGF- $\beta 1$. (C) Expression of mesenchymal-related proteins (a-SMA, FAP) and ECM-related protein (collagen 1) depending on treating with TGF- $\beta 1$ concentration in LX2 cells. (D) Intensity of a-SMA treated $10 \mu \mathrm{M}$ pirfenidone, $0.5 \mu \mathrm{M}$ or $1 \mu \mathrm{M}$ retinoic acid and forskolin with $20 \mathrm{ng} / \mathrm{ml}$ TGF- $\beta 1$ for $48 \mathrm{hr}$ in LX2 cells. (E-F) Expression of EMT-related protein (N-cadherin, Ecadherin, Snail) when LX2 cells were treated with 0.5 or $1 \mu \mathrm{M}$ of retinoic acid (E) or forskolin (F) with or without $20 \mathrm{ng} / \mathrm{ml}$ TGF- $\beta 1$ for 48 hr. All images were obtained using the Operetta CLS system. 

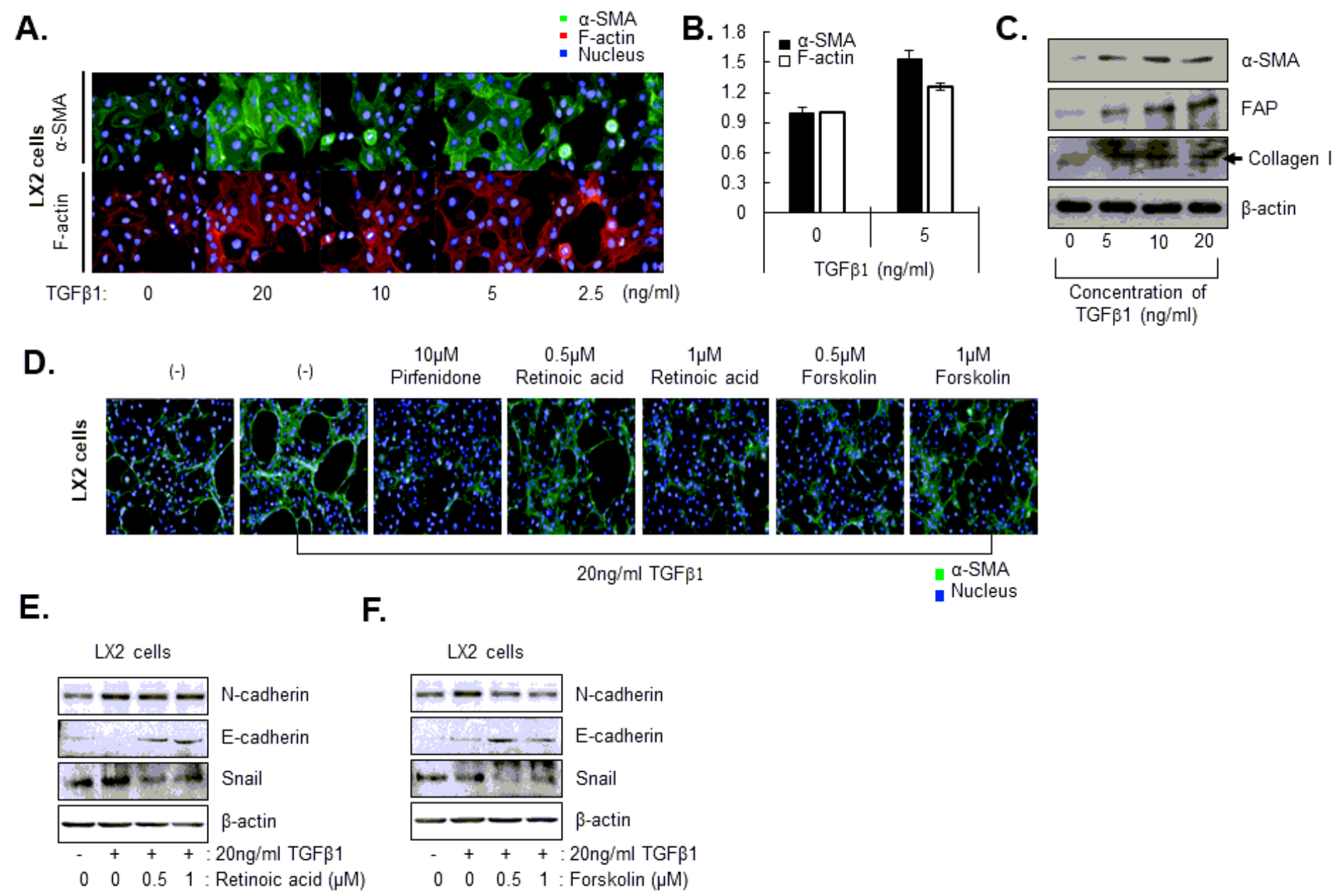

\section{Figure 3}

Anti-fibrosis effects of hit compounds in phenomic-based 2D culture of hepatic stellate cells. (A) Intensity of a-SMA and F-actin in hepatic stellate cells in a 2D culture system treated with TGF- $\beta 1$. Cells were treated with 2.5, 5, 10, or $20 \mathrm{ng} / \mathrm{ml}$ TGF- $\beta 1$ in $2 \%$ FBS media. (B) Calculated intensity of a-SMA and Factin when LX2 cells were treated with $5 \mathrm{ng} / \mathrm{ml}$ TGF- $\beta 1$. (C) Expression of mesenchymal-related proteins (a-SMA, FAP) and ECM-related protein (collagen 1) depending on treating with TGF- $\beta 1$ concentration in LX2 cells. (D) Intensity of a-SMA treated $10 \mu \mathrm{M}$ pirfenidone, $0.5 \mu \mathrm{M}$ or $1 \mu \mathrm{M}$ retinoic acid and forskolin with $20 \mathrm{ng} / \mathrm{ml}$ TGF- $\beta 1$ for $48 \mathrm{hr}$ in LX2 cells. (E-F) Expression of EMT-related protein (N-cadherin, Ecadherin, Snail) when LX2 cells were treated with 0.5 or $1 \mu \mathrm{M}$ of retinoic acid (E) or forskolin (F) with or without $20 \mathrm{ng} / \mathrm{ml}$ TGF- $\beta 1$ for 48 hr. All images were obtained using the Operetta CLS system. 

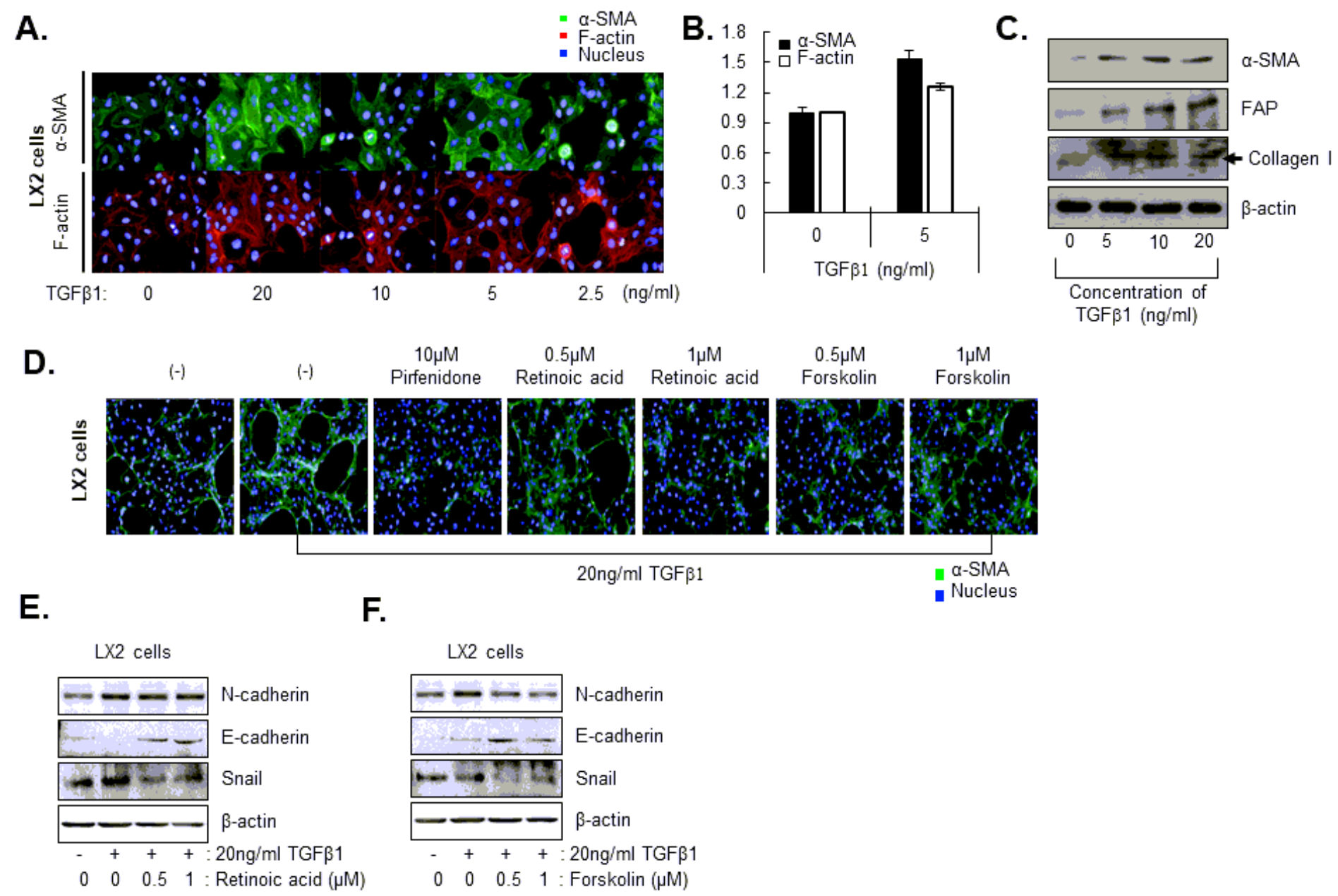

\section{Figure 3}

Anti-fibrosis effects of hit compounds in phenomic-based 2D culture of hepatic stellate cells. (A) Intensity of a-SMA and F-actin in hepatic stellate cells in a 2D culture system treated with TGF- $\beta 1$. Cells were treated with 2.5, 5, 10, or $20 \mathrm{ng} / \mathrm{ml}$ TGF- $\beta 1$ in $2 \%$ FBS media. (B) Calculated intensity of a-SMA and Factin when LX2 cells were treated with $5 \mathrm{ng} / \mathrm{ml}$ TGF- $\beta 1$. (C) Expression of mesenchymal-related proteins (a-SMA, FAP) and ECM-related protein (collagen 1) depending on treating with TGF- $\beta 1$ concentration in LX2 cells. (D) Intensity of a-SMA treated $10 \mu \mathrm{M}$ pirfenidone, $0.5 \mu \mathrm{M}$ or $1 \mu \mathrm{M}$ retinoic acid and forskolin with $20 \mathrm{ng} / \mathrm{ml}$ TGF- $\beta 1$ for $48 \mathrm{hr}$ in LX2 cells. (E-F) Expression of EMT-related protein (N-cadherin, Ecadherin, Snail) when LX2 cells were treated with 0.5 or $1 \mu \mathrm{M}$ of retinoic acid (E) or forskolin (F) with or without $20 \mathrm{ng} / \mathrm{ml}$ TGF- $\beta 1$ for 48 hr. All images were obtained using the Operetta CLS system. 

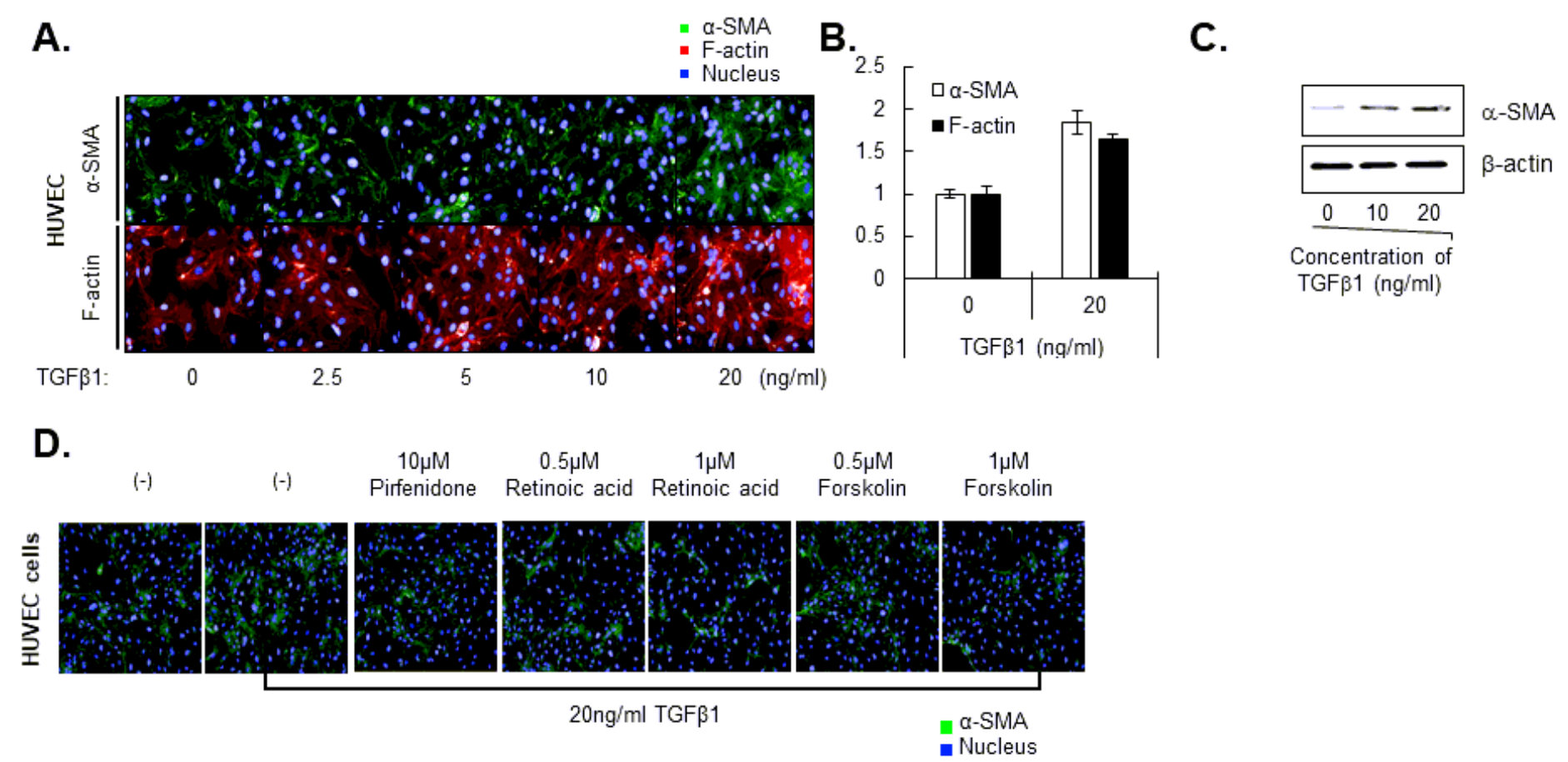

E.

F.
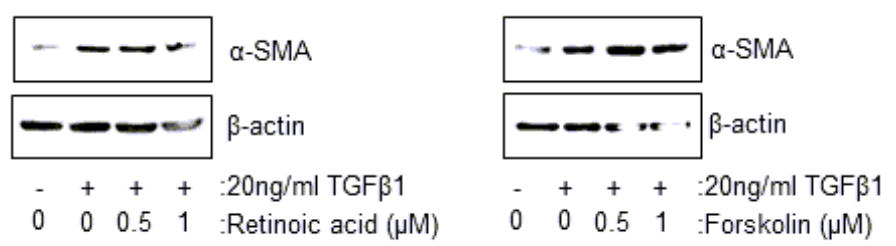

Figure 4

Anti-fibrosis effects of hit compounds in phenomic-based 2D assay of endothelial cells (HUVECs). (A) Intensity of a-SMA and F-actin in HUVEC cells in 2D culture system treating with TGF- $\beta 1$. Cells were treated with $2.5,5,10$, or $20 \mathrm{ng} / \mathrm{ml} \mathrm{TGF-} \beta 1$ (B) Calculated intensity of a-SMA and F-actin when HUVEC cells were treated with $20 \mathrm{ng} / \mathrm{ml} \mathrm{TGF- \beta 1}$ (C) Expression of mesenchymal-related protein ( $\mathrm{a}-\mathrm{SMA}$ ) depending on treating with TGF- $\beta 1$ concentration in HUVEC cells. (D) Intensity of a-SMA treated with 10 $\mu \mathrm{M}$ of pirfenidone, $0.5 \mu \mathrm{M}$ or $1 \mu \mathrm{M}$ of retinoic acid and forskolin with $20 \mathrm{ng} / \mathrm{ml}$ TGF- $\beta 1$ for $48 \mathrm{hr}$ in HUVEC cells. (E-F) Expression of a-SMA when HUVEC cells were treated with 0.5 or $1 \mu \mathrm{M}$ retinoic acid $(\mathrm{E})$ or forskolin (F). All images were obtained using the Operetta CLS system. 

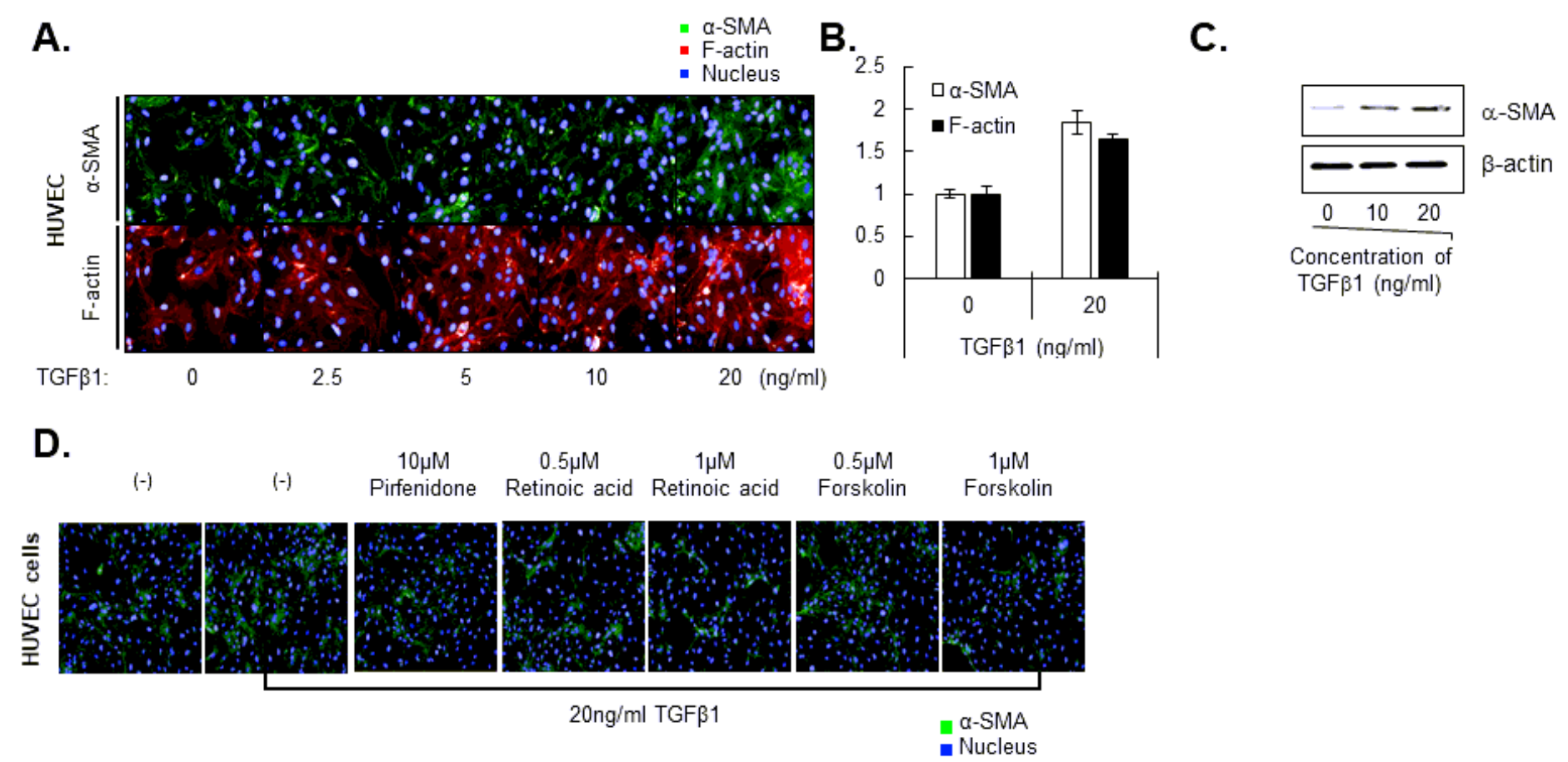

E.

F.
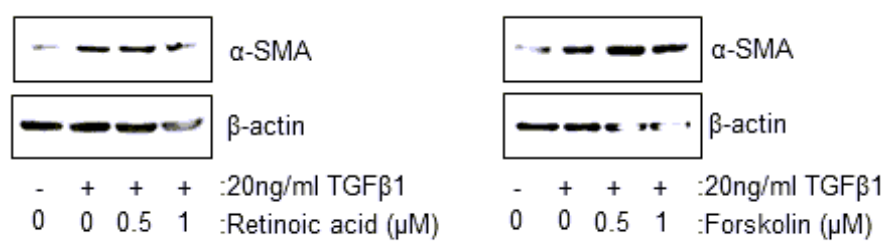

Figure 4

Anti-fibrosis effects of hit compounds in phenomic-based 2D assay of endothelial cells (HUVECs). (A) Intensity of a-SMA and F-actin in HUVEC cells in 2D culture system treating with TGF- $\beta 1$. Cells were treated with $2.5,5,10$, or $20 \mathrm{ng} / \mathrm{ml} \mathrm{TGF-} \beta 1$ (B) Calculated intensity of a-SMA and F-actin when HUVEC cells were treated with $20 \mathrm{ng} / \mathrm{ml} \mathrm{TGF- \beta 1}$ (C) Expression of mesenchymal-related protein ( $\mathrm{a}-\mathrm{SMA}$ ) depending on treating with TGF- $\beta 1$ concentration in HUVEC cells. (D) Intensity of a-SMA treated with 10 $\mu \mathrm{M}$ of pirfenidone, $0.5 \mu \mathrm{M}$ or $1 \mu \mathrm{M}$ of retinoic acid and forskolin with $20 \mathrm{ng} / \mathrm{ml}$ TGF- $\beta 1$ for $48 \mathrm{hr}$ in HUVEC cells. (E-F) Expression of a-SMA when HUVEC cells were treated with 0.5 or $1 \mu \mathrm{M}$ retinoic acid $(\mathrm{E})$ or forskolin (F). All images were obtained using the Operetta CLS system. 

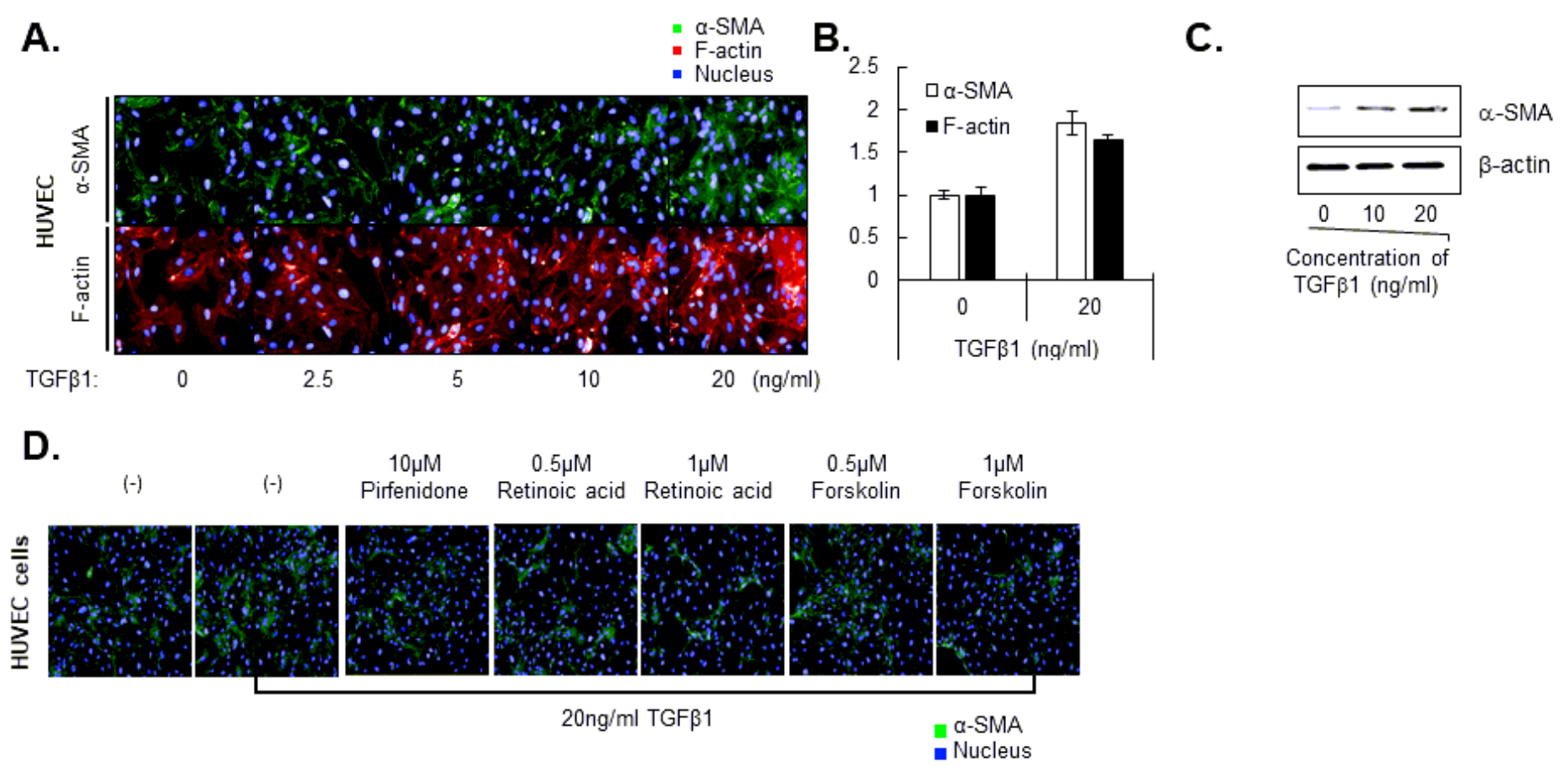

E.

F.
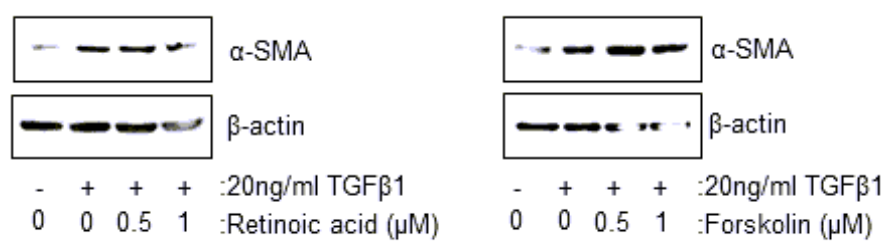

Figure 4

Anti-fibrosis effects of hit compounds in phenomic-based 2D assay of endothelial cells (HUVECs). (A) Intensity of a-SMA and F-actin in HUVEC cells in 2D culture system treating with TGF- $\beta 1$. Cells were treated with $2.5,5,10$, or $20 \mathrm{ng} / \mathrm{ml} \mathrm{TGF-} \beta 1$ (B) Calculated intensity of a-SMA and F-actin when HUVEC cells were treated with $20 \mathrm{ng} / \mathrm{ml} \mathrm{TGF- \beta 1}$ (C) Expression of mesenchymal-related protein ( $\mathrm{a}-\mathrm{SMA}$ ) depending on treating with TGF- $\beta 1$ concentration in HUVEC cells. (D) Intensity of a-SMA treated with 10 $\mu \mathrm{M}$ of pirfenidone, $0.5 \mu \mathrm{M}$ or $1 \mu \mathrm{M}$ of retinoic acid and forskolin with $20 \mathrm{ng} / \mathrm{ml}$ TGF- $\beta 1$ for $48 \mathrm{hr}$ in HUVEC cells. (E-F) Expression of a-SMA when HUVEC cells were treated with 0.5 or $1 \mu \mathrm{M}$ retinoic acid $(\mathrm{E})$ or forskolin (F). All images were obtained using the Operetta CLS system. 
A.

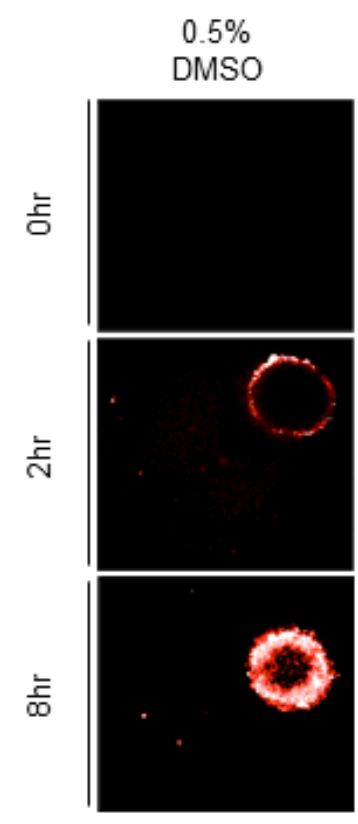

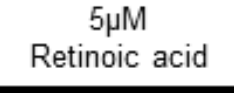
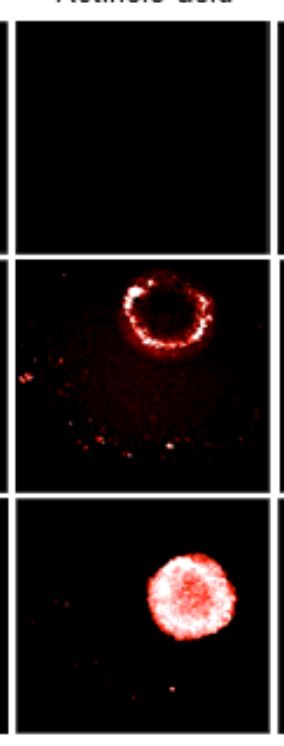

.

B.
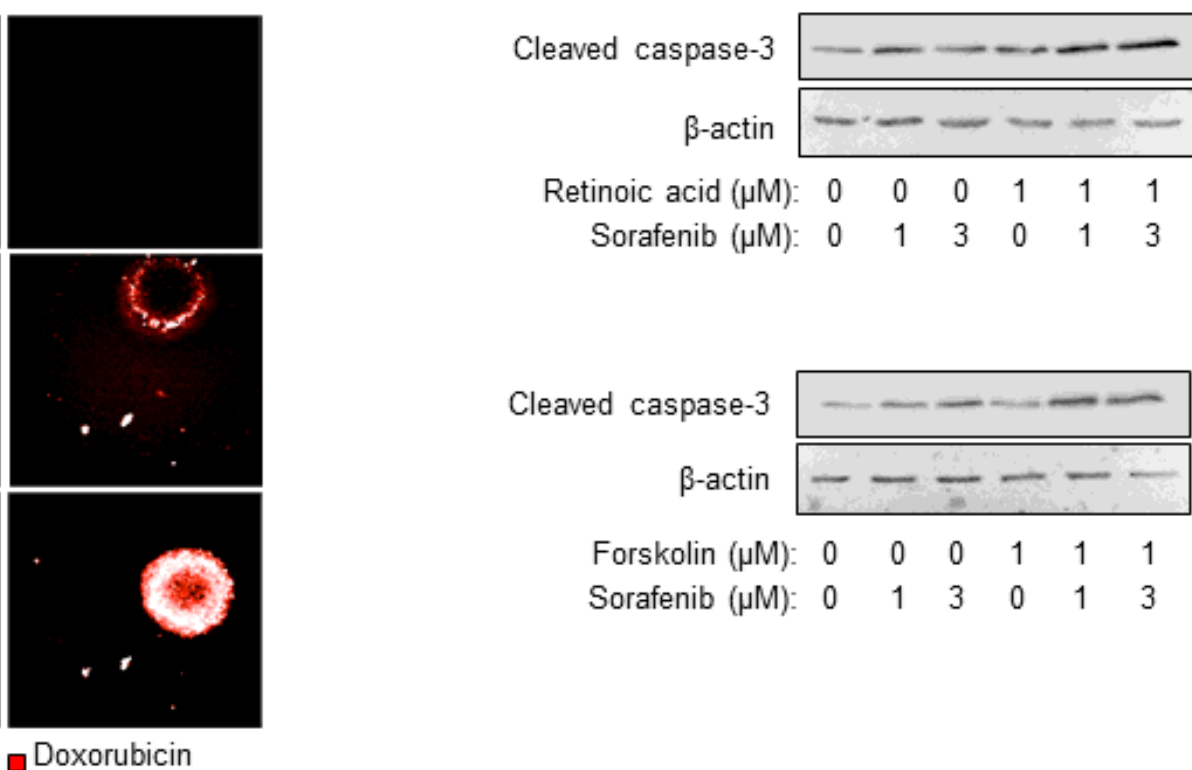

Cleaved caspase-3 $\beta$-actin

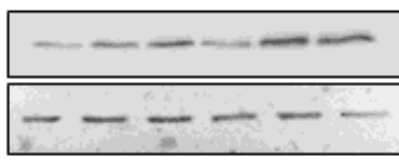

Forskolin $(\mu \mathrm{M}): \begin{array}{llllll}0 & 0 & 0 & 1 & 1 & 1\end{array}$ Sorafenib $(\mu \mathrm{M}): \begin{array}{llllll}0 & 1 & 3 & 0 & 1 & 3\end{array}$

\section{Figure 5}

Improvement of anti-cancer activity through combination with anti-fibrosis compounds. (A) Drug penetration of MCTS treated with $5 \mu \mathrm{M}$ retinoic acid or forskolin. After $48 \mathrm{hr}$ treatment with anti-fibrotic drugs, $10 \mu \mathrm{M}$ doxorubicin was added to cells for 0,2 , or $8 \mathrm{hr}$. (B) Expression of apoptosis-related protein, cleaved caspase-3, in MCTS with or without 1 or $3 \mu \mathrm{M}$ sorafenib and $1 \mu \mathrm{M}$ of retinoic acid or forskolin. All images were obtained using the Operetta CLS system. 
A.

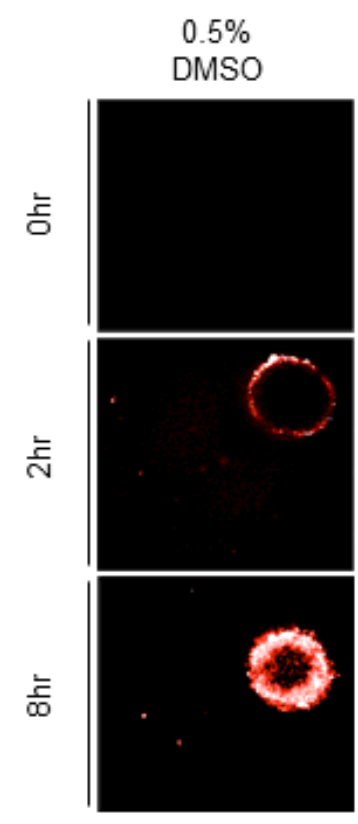

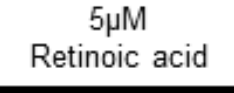
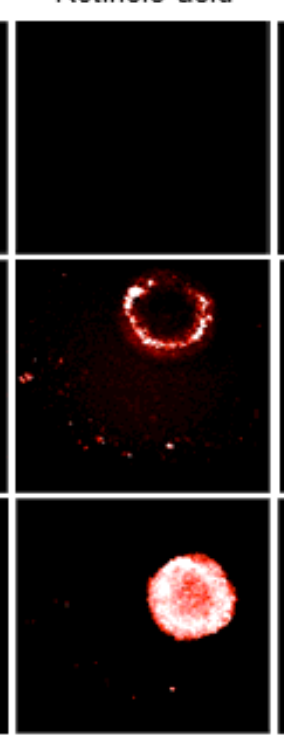

.

B.
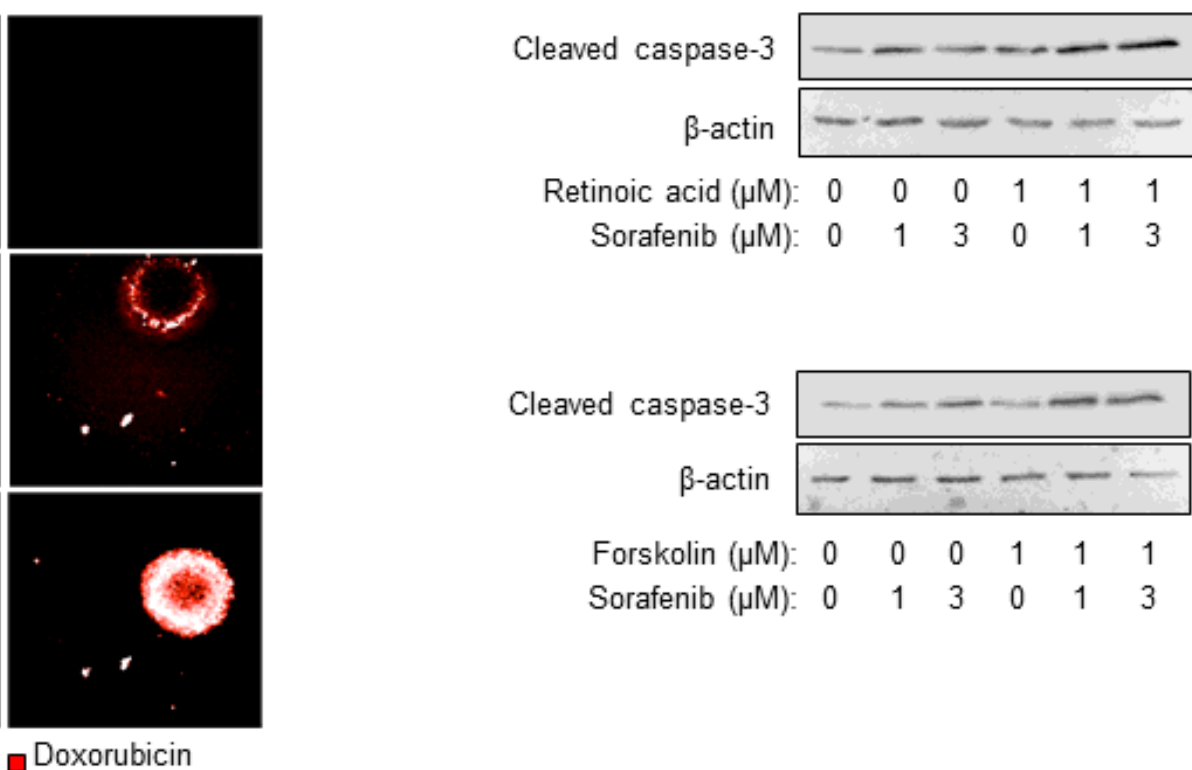

Cleaved caspase-3 $\beta$-actin

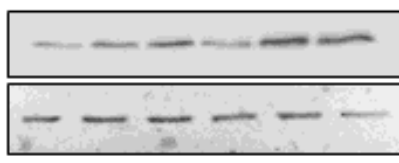

Forskolin $(\mu \mathrm{M}): \begin{array}{llllll}0 & 0 & 0 & 1 & 1 & 1\end{array}$ Sorafenib $(\mu \mathrm{M}): \begin{array}{llllll}0 & 1 & 3 & 0 & 1 & 3\end{array}$

\section{Figure 5}

Improvement of anti-cancer activity through combination with anti-fibrosis compounds. (A) Drug penetration of MCTS treated with $5 \mu \mathrm{M}$ retinoic acid or forskolin. After $48 \mathrm{hr}$ treatment with anti-fibrotic drugs, $10 \mu \mathrm{M}$ doxorubicin was added to cells for 0,2 , or $8 \mathrm{hr}$. (B) Expression of apoptosis-related protein, cleaved caspase-3, in MCTS with or without 1 or $3 \mu \mathrm{M}$ sorafenib and $1 \mu \mathrm{M}$ of retinoic acid or forskolin. All images were obtained using the Operetta CLS system. 
A.

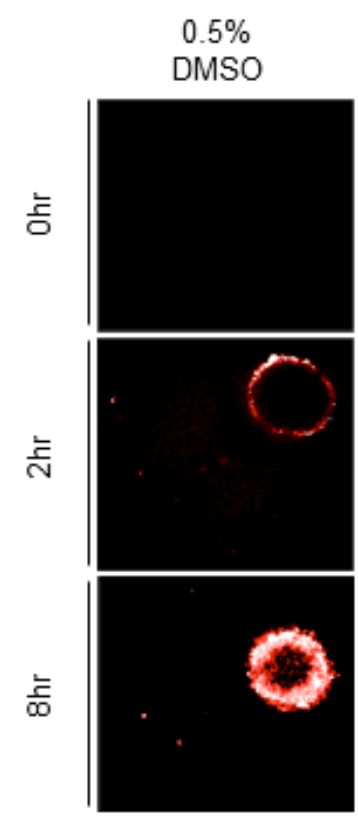

$5 \mu \mathrm{M}$

Retinoic acid
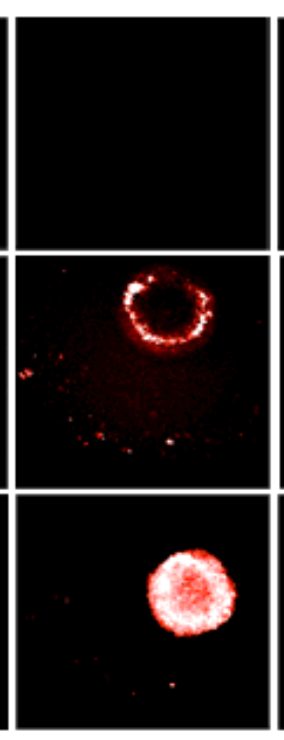

$5 \mu \mathrm{M}$

Forskolin

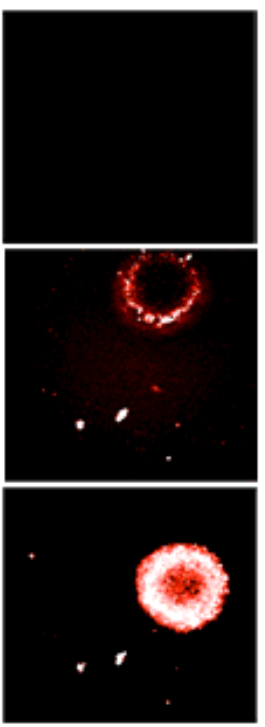

$\square$ Doxorubicin
B.

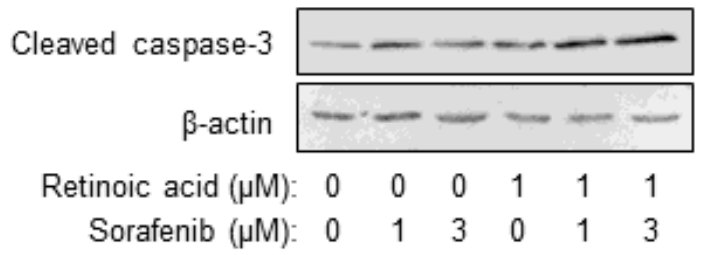

Cleaved caspase-3 $\beta$-actin

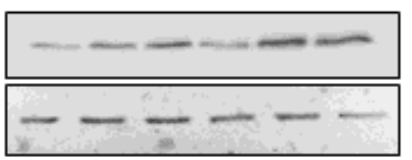

Forskolin $(\mu \mathrm{M}): \begin{array}{llllll}0 & 0 & 0 & 1 & 1 & 1\end{array}$ Sorafenib $(\mu \mathrm{M}): \begin{array}{llllll}0 & 1 & 3 & 0 & 1 & 3\end{array}$

\section{Figure 5}

Improvement of anti-cancer activity through combination with anti-fibrosis compounds. (A) Drug penetration of MCTS treated with $5 \mu \mathrm{M}$ retinoic acid or forskolin. After $48 \mathrm{hr}$ treatment with anti-fibrotic drugs, $10 \mu \mathrm{M}$ doxorubicin was added to cells for 0,2 , or $8 \mathrm{hr}$. (B) Expression of apoptosis-related protein, cleaved caspase-3, in MCTS with or without 1 or $3 \mu \mathrm{M}$ sorafenib and $1 \mu \mathrm{M}$ of retinoic acid or forskolin. All images were obtained using the Operetta CLS system.

\section{Supplementary Files}

This is a list of supplementary files associated with this preprint. Click to download.

- Supplementaryfigure.pdf

- Supplementaryfigure.pdf

- Supplementaryfigure.pdf 